Article

\title{
Impacts of Orbital and Constellation Parameters on the Number and Spatiotemporal Coverage of LEO-LEO Occultation Events
}

\author{
Congliang Liu $1,2,3,4, *$, Gottfried Kirchengast ${ }^{3,4}\left(\right.$ ) , Yueqiang Sun ${ }^{1,2,4}$, Veronika Proschek ${ }^{3,4}$, Xin Wang ${ }^{5}$, \\ Longfei Tian ${ }^{6}$, Qifei Du ${ }^{1,2,4}$, Weihua Bai ${ }^{1,2,4}$, Chunjun $\mathrm{Wu}^{1,4}$, Peng Hu ${ }^{1,4}$ and Guangyuan Tan ${ }^{1,4}$ \\ 1 National Space Science Center, Chinese Academy of Sciences (NSSC/CAS) and Beijing Key Laboratory of \\ Space Environment Exploration, Beijing 100190, China; syq@nssc.ac.cn (Y.S.); dqf@nssc.ac.cn (Q.D.); \\ baiweihua@nssc.ac.cn (W.B.); wuchunjun@nssc.ac.cn (C.W.); hupeng@nssc.ac.cn (P.H.); \\ tanguangyuan16@mails.ucas.ac.cn (G.T.) \\ 2 University of Chinese Academy of Sciences, Beijing 100190, China \\ 3 Wegener Center for Climate and Global Change (WEGC) and Institute for Geophysics, Astrophysics, \\ and Meteorology/Institute of Physics, University of Graz, 8010 Graz, Austria; \\ gottfried.kirchengast@uni-graz.at (G.K.); veronika.proschek@uni-graz.at (V.P.) \\ 4 Joint Laboratory on Occultations for Atmosphere and Climate (JLOAC) of NSSC/CAS, Beijing 100190, China, \\ and University of Graz, 8010 Graz, Austria \\ 5 Institute of Atmospheric Physics, Chinese Academy of Sciences, Beijing 100029, China; \\ wangx2003@mail.iap.ac.cn \\ 6 Innovation Academy for Microsatellite of Chinese Academy of Sciences, Shanghai 201210, China; \\ tianlf@microsate.com \\ check for \\ * Correspondence: 1cl@nssc.ac.cn; Tel.: +86-010-6255-7975
} updates

Citation: Liu, C.; Kirchengast, G.; Sun, Y.; Proschek, V.; Wang, X.; Tian, L.; Du, Q.; Bai, W.; Wu, C.; Hu, P.; et al. Impacts of Orbital and Constellation Parameters on the Number and Spatiotemporal Coverage of LEO-LEO Occultation Events. Remote Sens. 2021, 13, 4849. https://doi.org/10.3390/rs13234849

Academic Editor:

Nereida Rodriguez-Alvarez

Received: 23 October 2021

Accepted: 25 November 2021

Published: 29 November 2021

Publisher's Note: MDPI stays neutral with regard to jurisdictional claims in published maps and institutional affiliations.

Copyright: (C) 2021 by the authors Licensee MDPI, Basel, Switzerland. This article is an open access article distributed under the terms and conditions of the Creative Commons Attribution (CC BY) license (https:/ / creativecommons.org/licenses/by/ $4.0 /)$.
Abstract: The development of small-satellite technologies allows the low Earth orbit intersatellite link (LEO-LEO) occultation method to observe the Earth's atmosphere with global coverage and acceptable costs using electromagnetic signals, in which the $\mathrm{L} / \mathrm{X} / \mathrm{K} / \mathrm{M}$ band and short-wave infrared band signals have been well demonstrated to be suitable. We hence need to investigate the impacts of orbital and constellation parameters on the number and spatiotemporal distribution of LEOLEO occultation events for best-possible LEO-LEO occultation mission design and optimization at the targeted mission size. In this study, firstly, an occultation events location simulation model accounting for the right ascension of the ascending node (RAAN) precession was set up and the concept of a time-dependent global coverage fraction of occultation events was defined. Secondly, numerical experiments were designed to investigate the orbital parameters' impacts and to assess the performance of LEO-LEO occultation constellations, in which the Earth is divided into $5^{\circ} \times 5^{\circ}$ latitude and longitude cells. Finally, the number, timeliness, and global coverage fraction of occultation events for two-orbit and multi-orbit LEO-LEO constellations were calculated and analyzed. The results show that: (1) the orbit inclination and RAAN are the main impacting parameters followed by orbital height, while the RAAN precession is a relevant modulation factor; (2) co-planar counter-rotating receiving and transmitting satellite orbits are confirmed to be ideal for a two-satellite LEO-LEO constellation; (3) polar and near-polar orbit constellations most readily achieve global coverage of occultation events; near-equator orbit constellations with supplementary receiving and transmitting satellite orbit planes also readily form the occultation event geometry, though the occultation events are mainly distributed over low and low-to-middle latitude zones; and (4) a well-designed larger LEO-LEO occultation constellation, composed of 36-72 satellites, can meet the basic requirements of global numerical weather prediction for occultation numbers and timeliness, yielding 23,000-38,000 occultation events per day and achieving $100 \%$ global coverage in $12-18 \mathrm{~h}$.

Keywords: LEO-LEO occultation; occultation event numbers; spatiotemporal coverage; orbit elements; constellation design 


\section{Introduction}

Occultation is a well-proven remote sensing method that requires at least one electromagnetic signal transmitting satellite $(\mathrm{Tx})$ and one receiving satellite $(\mathrm{Rx})$ to observe the Earth's atmospheric thermodynamic state variables (e.g., pressure, temperature, and humidity) [1-6], chemical composition (e.g., the greenhouse gases $\mathrm{H}_{2} \mathrm{O}, \mathrm{CO}_{2}, \mathrm{CH}_{4}, \mathrm{~N}_{2} \mathrm{O}$, $\mathrm{O}_{3}$, and $\mathrm{CO}$ ) [7-11], dynamics (e.g., line-of-sight wind speed) [12], and ionospheric electron density profiles by using particular frequency signals [13]. Occultation events happen when the Tx and Rx see each other through the atmosphere just before (a setting occultation event) or after (a rising occultation event) they are occulted by the Earth during the relative orbital motion [14]. The amount of occultation data depends on the odds of occultation events, so satellite orbit and constellation design is significant to occultation Earth observation missions to optimize the performances and reduce the costs.

In recent years, Global Navigation Satellite System (GNSS) radio occultation (RO) has become a major method by which to observe the Earth's atmospheric thermodynamic state variables, but it has a temperature and humidity ambiguity problem in the troposphere [15-18]. Fortunately, as an advanced technique, low Earth orbit intersatellite link (LEO-LEO) microwave occultation (LMO), using various $\mathrm{X} / \mathrm{K}$ band and $\mathrm{M}$ band frequencies around the $22 \mathrm{GHz}$ and $183 \mathrm{GHz}$ water absorption lines, can exploit both the refraction and absorption of the signals to solve the temperature-humidity ambiguity problem; thus, LMO enables us to retrieve pressure, temperature, and humidity profiles without background information $[5,15]$. Liquid water and rain rate profiles as well as turbulence and complementary 3D cloud information can be co-observed [18,19]. Additionally, the LEO-LEO infrared laser occultation (LIO), using laser signals in the short-wave infrared band $(2-2.5 \mu \mathrm{m})$ between LEO satellites, has been designed to accurately observe key trace gas species for chemistry and the climate (e.g., the greenhouse gases $\mathrm{H}_{2} \mathrm{O}, \mathrm{CO}_{2}, \mathrm{CH}_{4}$, $\mathrm{N}_{2} \mathrm{O}, \mathrm{O}_{3}$, and $\mathrm{CO}$ ), line-of-sight wind speed, and profiles of cloud layers and aerosols as by-products $[7-9,18,20,21]$. LIO measures the absorption of the signals to retrieve volume mixing ratio (VMR) profiles of the greenhouse gases, in which the differential transmission principle between carefully selected pairs of absorption signals and reference signals is commonly used. The feasibility of the LMO and LIO techniques has been proven by ground-based demonstration experiments [10,11,17,18,22,23].

Nowadays, the development of small satellites and remote sensing instrument miniaturization technologies allows LEO-LEO occultation to provide observations with global coverage and acceptable mission costs. Moreover, the increase in the in-orbit and planned LEO constellations provides more signal and instrument onboard opportunities to realize space-borne LEO-LEO occultation observations in combination with other spacecraft, such as communication, navigation, and meteorological satellites. Therefore, the impacts of the $\mathrm{Rx}$ and Tx orbital parameters on the number and spatial-temporal distribution of LEO-LEO occultation events and the exploration capability of LEO-LEO occultation constellations need to be deeply investigated and assessed as they are significant to LEO-LEO mission design and optimization.

In this study, the impacts of the Rx and Tx orbital parameters on the LEO-LEO occultation event numbers and distribution were investigated and assessed using a two-satellite constellation with one LEO Rx and one LEO Tx based on simulation datasets by the variable separation approach. Then, $2 \mathrm{Rx} / 2 \mathrm{Tx}, 6 \mathrm{Rx} / 6 \mathrm{Tx}$, and $12 \mathrm{Rx} / 12 \mathrm{Tx}$ dual-orbit constellations and 6Rx/6Tx multiple-orbit constellations were designed and their occultation event numbers and spatial-temporal distributions were evaluated and analyzed.

This paper is organized as follows. Section 2 provides a brief review of relevant LEO orbital design studies. Section 3 describes the methods used in the LEO-LEO occultation event simulation and the constellation performance assessment. The design and results of the numerical experiment are described and analyzed in Section 4. The results and their application significance are discussed in Section 5, and some conclusions are drawn in Section 6. 


\section{Brief Review of Previous Occultation Mission and Orbit Design Studies}

The occultation concept was first proven by the Mariner V spacecraft during its Venus exploration in 1967 [24]. The first Earth observation occultation experiment using geostationary and LEO satellites was performed in 1974 [25]; however, at that time, the occultation technique developed very slowly because this type of constellation provided limited occultation data and many satellites were required for global coverage of the Earth, leading to unaffordable costs.

Decades later, with the emergence of the Global Positioning System (GPS) constellation, GPS RO was proposed to probe the Earth's atmosphere using GPS and LEO satellites (Tx and Rx, respectively). The GPS RO was demonstrated by the proof-of-concept GPS/Meteorology (GPS/MET) mission [3,26]. Subsequently, many GPS RO missions have been launched, including Challenging Minisatellite Payload (CHAMP) [27-29], Gravity Recovery and Climate Experiment-A (GRACE-A) [30,31], and Meteorological Operational Satellite-A/-B (MetOp-A/-B) [32], which are single Rx constellations, and the Constellation Observing System for Meteorology, Ionosphere, and Climate (COSMIC) [33-35], which is the first Rx constellation with six Rxs. In recent years, with the development of Global Navigation Satellite Systems (GNSSs), such as the Chinese BeiDou navigation satellite system (BDS), the European Galileo navigation satellite system (GALILEO), and the Russian GLObal NAvigation Satellite System (GLONASS), the new GNSS RO missions are commonly equipped with multi-GNSS RO receivers, such as the FengYun-3C/-3D/-3E GNSS Occultation Sounder (FY-3C/-3D/-3E GNOS) [36,37], the COSMIC II, and the spire weather constellation [38].

In the GNSS-LEO RO constellation design and exploration capability evaluation, the orbital parameters of the GNSS satellites are fixed and known, so only the orbital parameters of the LEO satellites need to be designed and analyzed for the observation of a particular geographic area or the whole Earth by a certain number and distribution of RO events. To obtain more GNSS RO events and a better spatial-temporal distribution for particular areas, several researches have been carried out and published. The impacts of LEO orbital and constellation parameters on the GNSS RO event number and global distribution have been investigated $[14,39,40]$. Practical LEO orbital parameters and constellations were designed and optimized for GNSS RO observations in particular regions, such as a tropical zone [41], the Asia Pacific region [42], and an area in Egypt [43,44]. Moreover, with the development of global and regional navigation satellite systems, the optimal design of LEO constellations for multi-GNSS RO missions has also been conducted [14,39].

Given that the importance of occultation and its data was well-proven by the GNSS $\mathrm{RO}$, a number of LEO-LEO missions, such as the Atmospheric Moisture and Ocean Reflection Experiment (AMORE) [4], the Water vapor and Temperature in the troposphere and Stratosphere (WATS) mission [45], the Active Temperature, Ozone, and Moisture Microwave Spectrometer (ATOMMS) [15], the Atmosphere and Climate Explorer Plus (ACE+) $[5,46-48]$, the Atmospheric Climate and Chemistry in the UTLS Region And climate Trends Explorer (ACCURATE) mission [20,49,50], and the Climate and Atmospheric Composition Exploring Satellites (CACES) mission, have been proposed [19]. The LMO concept was also translated to Mars in a mission concept called the Mars Atmospheric Climate Observatory (MACO), whose development began with seed funding from National Aeronautics and Space Administration (NASA) in 2000 [51]. An author group including the lead authors of this study has also conducted a detailed review of low Earth orbit occultation using microwave and infrared laser signals for monitoring the atmosphere and climate [18].

In the LEO-LEO occultation constellation design, the orbital parameters of both the $\mathrm{Rx}$ and $\mathrm{Tx}$ are unknown, which provides more free dimensionalities to optimize the occultation observation system but makes the constellation more complicated and difficult to design. On the other hand, LEO-LEO occultation is commonly considered to require many satellites to provide global coverage of the atmosphere, which leads to unaffordable costs, so there is currently no operational space-borne LEO-LEO constellation. Therefore, the 
number of studies on LEO-LEO occultation constellation design and evaluation, especially constellations with a large number of satellites, is less than that on GNSS RO. Specific simple LEO-LEO constellations with one pair or two pairs of Rx and Tx satellites for demonstration missions have been designed, in which the polar orbits are commonly used and the daily occultation event distribution along the latitude and longitude are illustrated by the use of maps and histograms [19]. Walker and 2D lattice flower constellations composed of a certain number of satellites for a LEO-LEO occultation mission have been proposed and evaluated [52].

\section{LEO-LEO Occultation Simulation and Assessment Methods}

\subsection{LEO-LEO Occultation Geometry Simulation Model}

As shown in Figure 1, during a LEO-LEO occultation active period, the transmitting signal ray path from the Tx first passes through the atmosphere and is captured by the $\mathrm{Rx}$; then, with the relative motion of the Tx and $R x$, the atmosphere is vertically scanned and remotely observed. A LEO-LEO occultation event occurs when the connecting line between the Tx and Rx and its tangent point with the atmospheric layers satisfy a certain geometric relationship. The mathematical criteria are: (1) the tangent point is between the Tx and the Rx; (2) the height of the tangent point is between the bottom and the top of the atmosphere; and (3) the azimuth angle of the Tx relative to the $\mathrm{Rx}^{\prime}$ s running direction is in a particular range.

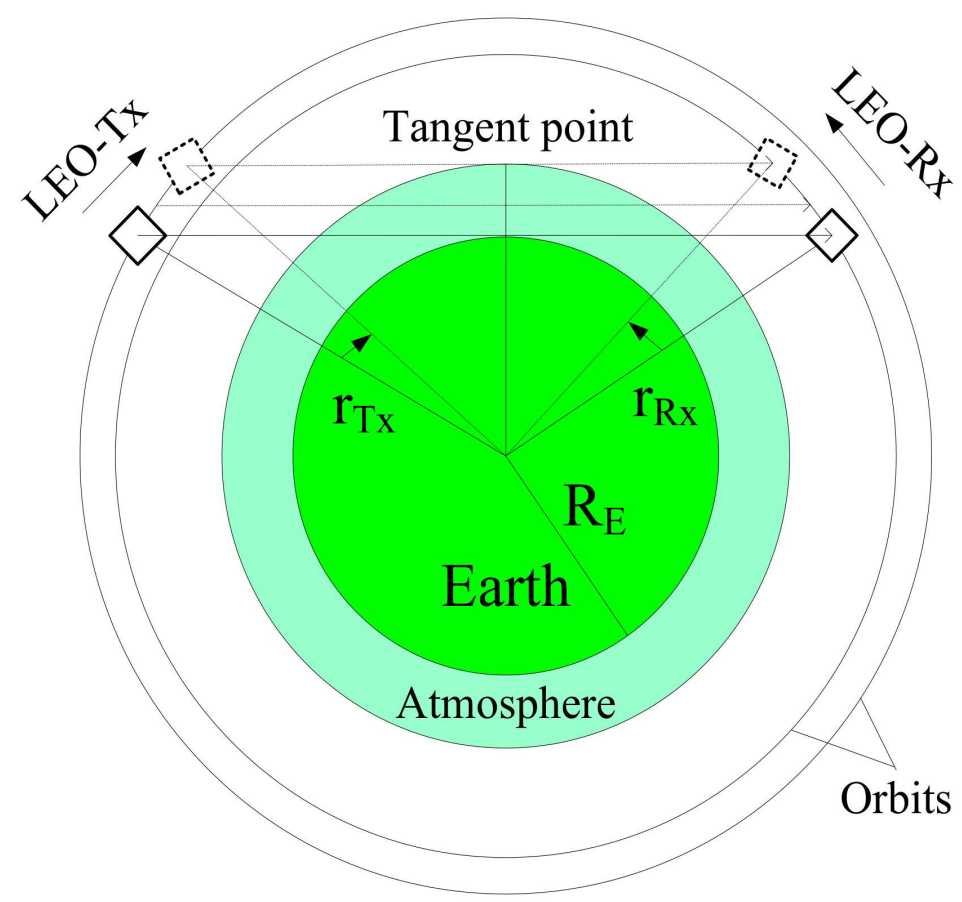

Figure 1. Occultation geometry between one LEO transmitting satellite (Tx) and one LEO receiving satellite $(\mathrm{Rx})$, schematically illustrating the signal ray path passing through the atmosphere and the tangent point of a rising occultation event. The solid-line squares and dotted-line squares represent the occultation event starting time (near-surface) and ending time (top-of-atmosphere) positions of the LEO-LEO satellite pair, respectively.

The unperturbed, two-body orbits are always conic sections; therefore, the Earth orbits are commonly described as an ellipse by Keplerian elements, i.e., eccentricity (e), semimajor axis (a), inclination (i), longitude of the ascending node $(\Omega)$, argument of periapsis (AP, $\omega)$, and true anomaly $(v)$. However, real Earth orbits have perturbations due to the gravitational pull of bodies other than the Earth, the nonsphericity of the Earth, atmospheric drag, relativistic effects, radiation pressure, electromagnetic forces, and so on. 
Therefore, in the simulation, $\mathrm{Rx}$ and Tx satellite positions and velocities were calculated from two-line element (TLE) sets through the simplified general perturbations4/simplified deep space perturbations-4 (SGP4/SDP4) model propagator, which takes the general and deep space orbital perturbations into account [53]. Among these perturbations, the right ascension of the ascending node (RAAN) precession is a key factor impacting the long-term LEO-LEO occultation event numbers and distributions, which can be described as Equation (1), and the real RAAN at epoch $t$ can be estimated by Equation (2) [54]:

$$
\begin{gathered}
\dot{\Omega}=-1.5 J_{2} n\left(R_{E} /\left(a-a \cdot e^{2}\right)\right)^{2} \cos i \\
\Omega=\Omega_{0}-\left(\dot{\Omega}_{E}-\dot{\Omega}\right)\left(t-t_{0}\right)
\end{gathered}
$$

In these equations, the constant $J_{2}=1.08262668 \times 10^{-3}$ is a zonal coefficient according to the Joint Earth Gravity Model 3 (JGM-3) geopotential model [54], $n$ is the angular velocity, $a$ denotes the semi major axes, $e$ is the eccentricity, $R_{E}=6378 \mathrm{~km}$ is the Earth's equatorial radius, and $\Omega_{0}, \dot{\Omega}_{E}$, and $\dot{\Omega}$ are the RAAN at the reference time $t_{0}$, the angular velocity of the Earth's rotation, and the drift angular velocity of the RAAN, respectively.

From Equation (1), one can find that when the orbital inclination is $90^{\circ}$, the RAAN precession angular velocity is 0 , the prograde orbit RAAN precession angular velocity is greater than 0 , and the retrograde orbit RAAN precession angular velocity is less than 0 ; that is, the RAAN precession of the prograde and retrograde orbits proceeds in different directions. Additionally, the greater the difference between the orbit inclination and $90^{\circ}$, the greater the precession speed, while the higher the orbit altitude, the lower the precession speed.

All the possible ray paths connecting the Tx and Rx were considered by setting the sampling rate to $3 \mathrm{~s}$, which we found to be adequate to safely capture all events while at the same time affordable in terms of computing time for the large number of simulation cases. The signal propagation from $\mathrm{Tx}$ to $\mathrm{Rx}$ was approximated by a straight line, which is reasonable and adequate since the atmospheric bending is very small and can be neglected in such occultation event number and distribution analyses [41]. The atmosphere altitude range was set from 0 to $120 \mathrm{~km}$, i.e., from the ground surface to the top of the neutral atmosphere. The location, i.e., the latitude, longitude, and altitude of the LEO-LEO occultation tangent point, was calculated according to [54]:

$$
\begin{gathered}
r_{T R}=r_{T x}-r_{R x} \\
\hat{n}_{T R}=\left(r_{T R}\right) /\left\|r_{T R}\right\| \\
R_{T}=\left\|r_{T x} \cdot \hat{n}_{T R}\right\| \\
r_{T P}=r_{T x}-\hat{n}_{T R} \cdot R_{T}=\left(X_{T P}, Y_{T P}, Z_{T P}\right) \\
\left\{\begin{array}{l}
\varphi_{T P}=\arctan \left(Z_{T P}\left(N+h_{T P}\right) / \sqrt{X_{T P}^{2}+Y_{T P}^{2}} /\left(N \cdot\left(1-e_{E}^{2}\right)+h_{T P}\right)\right) \\
\lambda_{T P}=\arctan \left(X_{T P} / Y_{T P}\right) \\
h_{T P}=Z_{T P} / \sin \varphi_{T P}-N \cdot\left(1-e_{E}^{2}\right) \\
N=a_{E} / \sqrt{1-e_{E}^{2} \sin ^{2} \varphi_{T P}} \\
\varphi_{T P 0}=\arctan \left(Z_{T P} / \sqrt{X_{T P}^{2}}+Y_{T P}^{2}\right) \\
\left|\varphi_{T P i+1}-\varphi_{T P i}\right| \leq 10^{-12}
\end{array}\right.
\end{gathered}
$$


where $r_{T R}$ is the vector from $\mathrm{Rx}$ to $\mathrm{Tx}, r_{T x}$ and $r_{R x}$ are the position vectors of $\mathrm{Tx}$ and $\mathrm{Rx}$ respectively, $\hat{n}_{R T}$ is the unit vector along the direction from $R x$ to $T x, R_{T}$ is the projection of the Tx position vector on the Rx to Tx connecting line, $r_{T P}$ is the position vector of the occultation tangent point, $\left(X_{T P}, Y_{T P}, Z_{T P}\right)$ are the three-dimensional coordinates of the occultation tangent point in the Earth-centered Earth-fixed (ECEF) coordinate system, $a_{E}$ is the equatorial radius of the Earth, $e_{E}$ is the first numerical eccentricity of the ellipsoid, $N$ is the distance from the surface back to the Z-axis intersection along the ellipsoid normal, and $\varphi_{T P}, \lambda_{T P}$, and $h_{T P}$ are the latitude, longitude, and height of the occultation tangent point in the geodetic coordinate system, respectively, where $\varphi_{T P}$ needs to be solved by iteration.

The azimuth angle of the Tx relative to the Rx running direction ranges were set as $0-40^{\circ}, 320-360^{\circ}$ for rising occultation events and $140-220^{\circ}$ for setting occultation events, which seems reasonable since, when the Tx satellite is located in these azimuth ranges, the satellite's attitudes will be adjusted to make sure that the Tx and Rx beams point to each other. The elevation angle $E$ and the azimuth angle $A$ of the Tx relative to the $\mathrm{Rx}$ running direction are defined in a Cartesian coordinate system with the Rx mass center as the origin,

$$
\begin{gathered}
\left\{\begin{array}{l}
x=v_{R x} \\
y=v_{R x} \times r_{R x} \\
z=x \times y
\end{array}\right. \\
\sin E=z \cdot r_{T R} /\left(\|z\| \cdot\left\|r_{T R}\right\|\right) \\
r_{A}=r_{T R}-\left\|r_{R T}\right\| \cdot \sin E \cdot z /\|z\| \\
\cos A=r_{A} \cdot x /\left(\left\|r_{A}\right\| \cdot\|x\|\right)
\end{gathered}
$$

where $v_{R x}$ is the Rx velocity vector and $r_{A}$ is the projection of $r_{T R}$ in the xy coordinate plane.

\subsection{Global Coverage Fraction of Occultation Events}

The Earth's surface is divided into spatial grid cells according to a certain rule, and the ratio of the accumulated area of grid cells visited by occultation events to the total area of the grid (i.e., the global surface area of the Earth) is defined as the global coverage fraction (GCF) of occultation events, which can be described by

$$
G C F=\left(A_{o c c} / A_{E}\right) \times 100 \%
$$

where $A_{o c c}$ indicates the accumulated area of the grid cells visited by occultation events and $A_{E}$ represents the total grid area, i.e., the entire Earth's surface in the given case of a global coverage fraction (for a prescribed regional coverage fraction, e.g., for the country of China only, one would use the entire area of the country as a reference).

To quantitatively assess the observation efficiency of the LEO-LEO constellations, occultation events were simulated and an event ID number, latitude, longitude, time, and some other accessary information were stored in an occultation table for statistical analysis. The specific analysis process is as follows: (1) accumulate the occultation events in the study period in the corresponding geographic grids according to the location and time information; (2) calculate the global coverage fraction of the occultation events using the above method; and (3) conduct a quantitative analysis of the spatiotemporal distribution of occultation events for specific constellations. Since the locations of occultation events are commonly marked with longitude and latitude, the entire Earth was divided into 
$25925^{\circ} \times 5^{\circ}$ grid cells according to latitude and longitude in this study. This density of gridding was found to be suitable for our purpose of globally illustrating geographical patterns of GCFs, without making the cell resolution too fine-grained, so that the cells receive robust coverage by occultation events for our LEO-LEO study design.

\section{Experimental Results and Analysis}

\subsection{Impacts of the Orbital Parameters on LEO-LEO Occultation Events}

4.1.1. Orbital Parameter Setup for the Two-Satellite Experimental Demonstration Mission

Based on a pair of Rx and Tx satellites, the impacts of the orbital parameters on the number and spatial-temporal distribution of LEO-LEO occultation events were analyzed using the variable separation approach, in which the analyzed target parameter was set as a discrete variable while other parameters were fixed as certain values. Commonly, near-circular orbits are adopted for the LEO Rx. In this case, the impact of the orbital eccentricity on the occultation events is small for typical circular-orbit eccentricities of order 0.0001 in real cases (e.g., near 0.0005 in the case of the International space station). The eccentricity of both the Rx and Tx orbits was hence fixed at 0.0001 and this parameter not further analyzed. The true anomaly is a constantly changing variable with time, while the impact of the initial mean anomalies (M0) on the two-satellite constellation occultation events is very small; therefore, the M0 of the Rx and Tx orbits can be set as arbitrary values and here they were fixed at $210^{\circ}$ and $30^{\circ}$, respectively.

The inclination, RAAN, and AP are three elements defining the orientation of the orbital plane in which the Rx and Tx orbits are embedded, while the semi major axis (a) defines the size of the orbits and strongly relates to the satellite's height and velocity. Hence, there are in principle four key orbital parameters for LEO-LEO occultation orbital and constellation design. Since our test experiments indicated that, for the near-circular orbits, the impact of AP on the number of LEO-LEO occultation events is very small, the AP of both the Rx and Tx orbits was fixed at $80^{\circ}$ and this parameter not further analyzed.

In the numerical study, three experimental groups named ' $i$ ', 'raan', and ' $h$ ', using lowercase letters to represent the research target parameters of inclination, RAAN, and height, respectively, were set up. The Rx and Tx orbital elements named ' $I$ ', 'RAAN', 'H', ' $A P^{\prime}$, and ' $E$ ' use uppercase letters instead, and the setups of the orbital parameters are shown in Table 1. The inclinations of $\mathrm{Rx}$ and Tx orbits were set to discrete values from $10^{\circ}$ to $170^{\circ}$ with a $10^{\circ}$ step length for group ' $\mathrm{i}$ ' and fixed values at $90^{\circ} / 90^{\circ}$ for the other groups. The RAANs of Rx and Tx orbits were set to discrete values from $30^{\circ}$ to $360^{\circ}$ with a $30^{\circ}$ step length for group 'raan' and fixed values at $180^{\circ} / 0^{\circ}$ for the other groups. The heights of the $\mathrm{Rx}$ and Tx orbits were set to discrete values from $400 \mathrm{~km}$ to $1300 \mathrm{~km}$ with a $100 \mathrm{~km}$ step length for group ' $h$ ' and fixed values at $500 \mathrm{~km} / 600 \mathrm{~km}$ for the other groups.

Table 1. The main orbital elements of the transmitting and receiving satellites for a two-satellite experimental constellation. The chosen set of values for some elements is denoted in the format of StartValue:Step:EndValue; simulations were performed using an ensemble of configurations stepping through all value settings for these elements.

\begin{tabular}{|c|c|c|c|}
\hline \multirow{2}{*}{$\begin{array}{c}\text { Orbit } \\
\text { Elements } \\
(\mathrm{Rx} / \mathrm{Tx})\end{array}$} & \multicolumn{3}{|c|}{ Experimental Groups } \\
\hline & i & raan & h \\
\hline I $\left(^{\circ}\right)$ & 10:10:170/10:10:170 & $90 / 90$ & $90 / 90$ \\
\hline RAAN $\left(^{\circ}\right)$ & $180 / 0$ & 30:30:360/30:30:360 & $180 / 0$ \\
\hline H (km) & $500 / 600$ & $500 / 600$ & 400:100:1300/400:100:1300 \\
\hline $\mathrm{AP}\left({ }^{\circ}\right)$ & $80 / 80$ & $80 / 80$ & $80 / 80$ \\
\hline Mo $\left({ }^{\circ}\right)$ & $210 / 30$ & $210 / 30$ & $210 / 30$ \\
\hline E & $0.0001 / 0.0001$ & $0.0001 / 0.0001$ & $0.0001 / 0.0001$ \\
\hline
\end{tabular}


It should be noted that the inclination of the Rx and Tx orbits was set to $90^{\circ}$ for the 'raan' and ' $h$ ' groups to avoid the RAAN precession having an influence on the simulation results; however, except for the $90^{\circ}$ case, the simulation results of the ' $i$ ' group were affected by both the inclination and the RAAN precession. Since the RAAN precession is slow and its impact on the short-term occultation event number and spatial-temporal distribution is not obvious, a long-term series of three months (90 days) of occultation events were simulated for each experimental group.

\subsubsection{Overall Simulation Results}

The variation in the 3-month LEO-LEO occultation event number with the Rx and Tx orbital elements of inclination, RAAN, and height is shown in Figure 2. One can see from Figure 2 that the 3-month occultation number changes strongly with the variation in the inclination and RAAN, but changes slightly with the variation in height for the Rxi90-Txi90 case. The impact of each of these orbital elements will be analyzed separately.

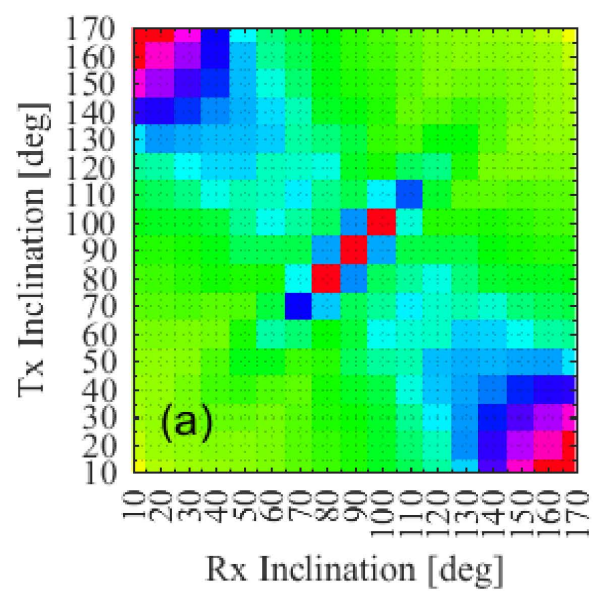

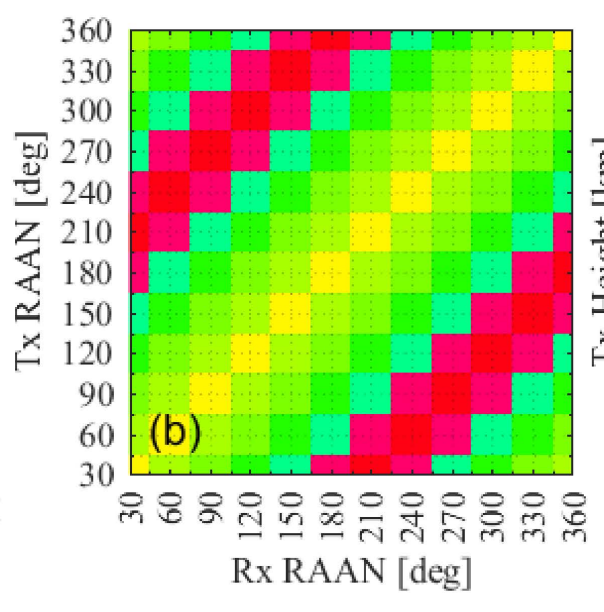

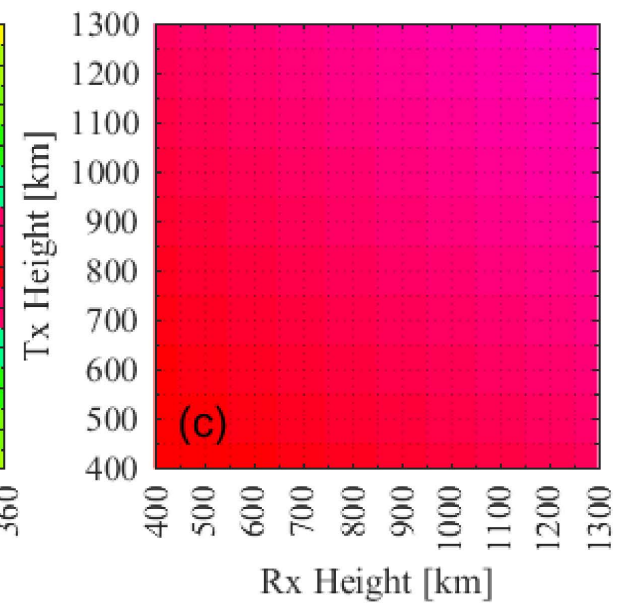

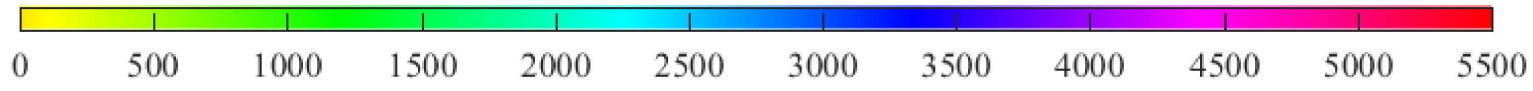

Figure 2. The variation in the 3-month accumulated LEO-LEO occultation event number (color scale) with the Rx and Tx orbital elements of inclination (a), RAAN (b), and height (c).

\subsubsection{Impact of the Inclination}

As shown in Figure 2a, the number of 3-month occultation events varies with the Rx and Tx orbital inclination greatly in the range 0-5500 and, accordingly, the orbits with an inclination between $65^{\circ}$ and $115^{\circ}$ were defined as 'near-polar orbits' (NPOs), the orbits with an inclination in the $0-45^{\circ}$ and $135-180^{\circ}$ intervals were defined as 'near-equator orbits' (NEOs), and orbits with an inclination in other intervals were defined as 'mediuminclination orbits' (MIOs). For the NPO constellations, when the inclinations of the Rx and Tx orbits are equal, the number of occultation events has a peak area, and the peak width is about $30^{\circ}$; when they are supplementary, the number of occultation events is reduced obviously. The NEO constellations also have an occultation event number peak area when the inclinations of the Rx and Tx orbits are supplementary, and the peak width is about $40^{\circ}$. However, the MIO constellations do not have an occultation event number peak area.

To further explore the spatial-temporal distribution of the NPO, NEO, and MIO constellation occultation events and the reason why the 3-month occultation numbers are different, Figures 3 and 4 show the global coverage and the occultation number trend beside the GCF trend of the occultation events for nine representative constellations, i.e., Rxi90Txi90, Rxi100-Txi100, Rxi80-Txi100, Rxi60-Txi60, Rxi120-Txi120, Rxi120-Txi60, Rxi40-Txi40, Rxi140-Txi40, and Rxi170-Txi10. 


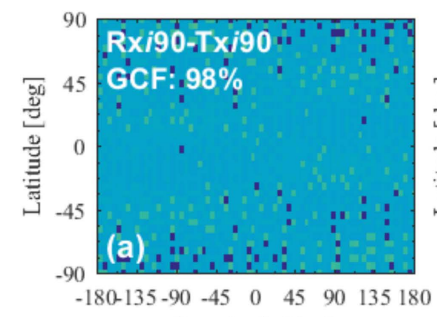

Longitude [deg]

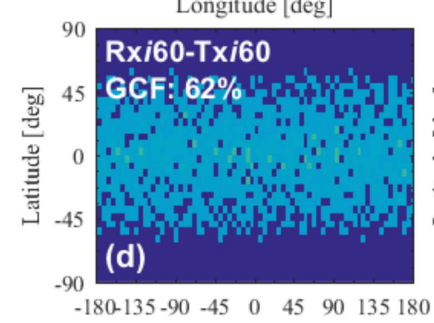

Longitude [deg]

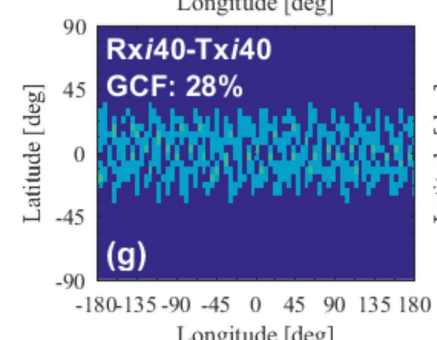

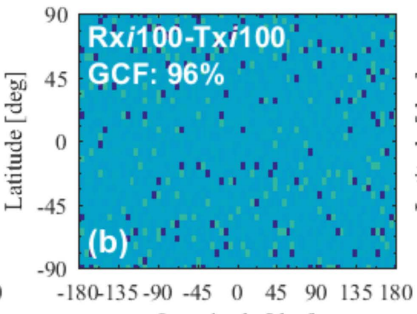

Longitude [deg]

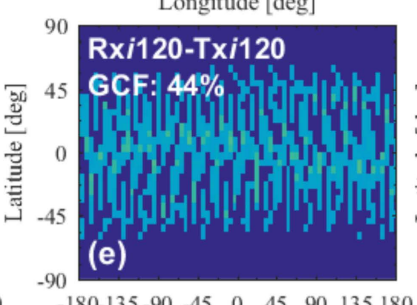

Longitude [deg]

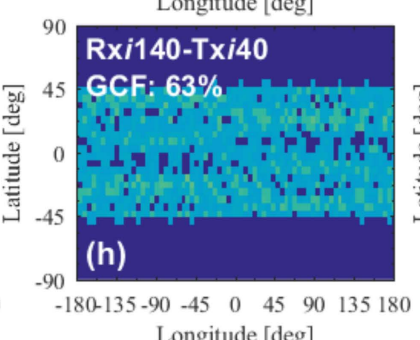

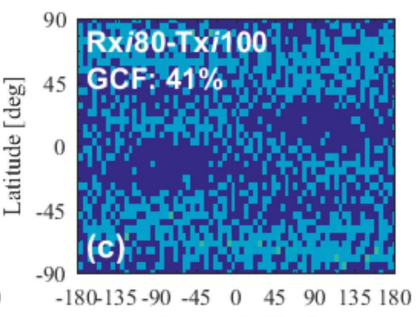

Longitude [deg]

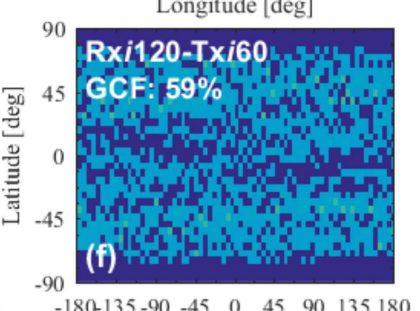

Longitude [deg]

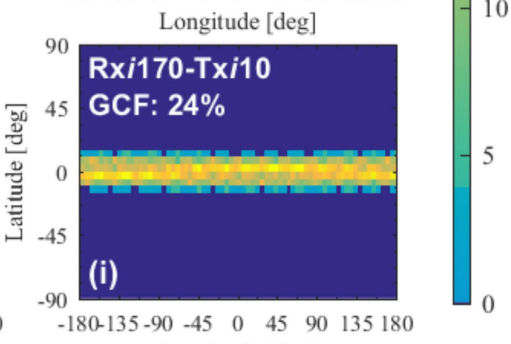

Longitude [deg]

Figure 3. The global coverage of LEO-LEO occultation events for representative constellations composed of a Rx satellite and a Tx satellite with particular orbital inclinations, illustrating the occultation numbers per $5^{\circ} \times 5^{\circ}$ latitude and longitude grid cell, while the GCFs were calculated using grid cell areas according to Equation (12).

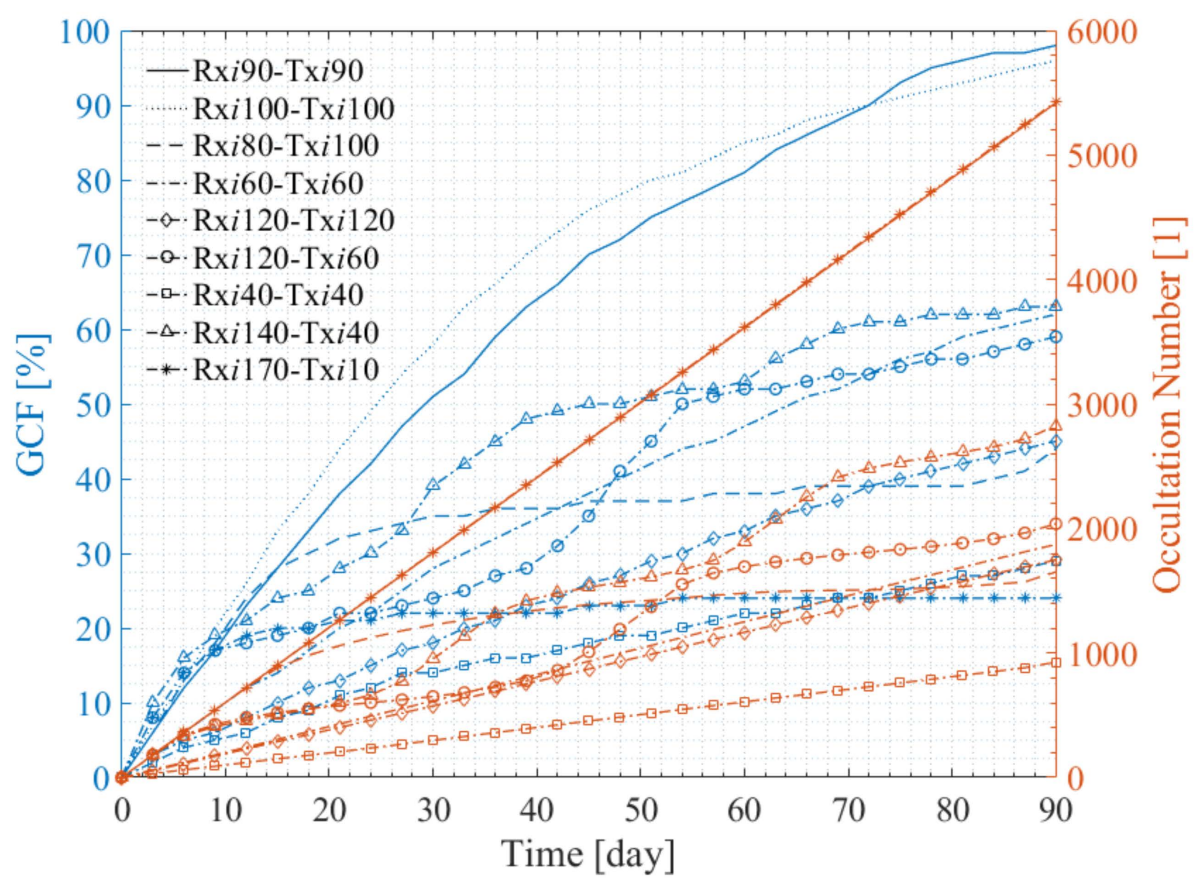

Figure 4. The accumulated time dependence of the LEO-LEO occultation event number (orange lines) and the global coverage fraction (GCF) (blue lines) for the representative LEO-LEO constellations composed of a Rx satellite and a Tx satellite with particular orbital inclinations. 
As shown in Figure 3a-c, the NPO constellation easily provides global coverage of occultation events, and the 3-month occultation global coverage fractions of Rxi90-Txi90, Rxi100-Txi100, and Rxi80-Txi100 are 98\%, 96\%, and 41\%, respectively. Comparing (a,b) with (c), one can see that the occultation number and the global coverage fractions of the NPO constellations with the same Tx and Rx inclination are larger than those of the NPO constellations with supplementary Tx and Rx inclinations. As shown in Figure $3 d-f$, the occultation events of the MIO constellation are mainly distributed in the low and middle latitude zones, and the global coverage fractions are relatively low. Comparing $(\mathrm{d}, \mathrm{e})$ with $(\mathrm{f})$, one can see that the occultation number and the global coverage fractions of the MIO constellations with the same Tx and Rx inclination are relatively small. As shown in Figure 3g-i, the occultation events of the NEO constellation are mainly distributed in the low latitude zone, and the global coverage fractions are relatively low. Comparing (g) with $(\mathrm{h}, \mathrm{i})$, the NEO constellations with the same Tx and Rx inclination have a small occultation number and a small global coverage fraction, while the NEO constellations with supplementary $\mathrm{Tx}$ and $\mathrm{Rx}$ inclinations have a large occultation number but very small global coverage fractions. With the decrease in the dihedral angle between the LEO plane and the equatorial plane, the global coverage fraction decreases, and the global coverage fraction of Rxi170-Txi10 is only $24 \%$.

Figure 4 shows the occultation number trend beside the global coverage fraction trend with time for the constellations shown in Figure 3. One can see that the occultation number of those constellations with the same Tx and Rx inclination increases in proportion to the time, while, except for in the Rxi170-Txi10 case, the constellations with supplementary Tx and $R x$ inclinations increase nonlinearly, and the occultation numbers grow very slowly during certain periods. The nonlinear characteristics of the occultation number and the GCF curves are caused by the RAAN precession, which will be analyzed in Section 4.2.

\subsubsection{Impact of the RAAN}

As shown in Figure $2 b$, for the polar orbit constellation, the number of 3-month occultation events varies with the Rx and Tx RAAN greatly in the range 0-5500, which is mainly related to the RAAN difference of the Rx and the Tx. When the RAAN difference of the $R x$ and $T x$ is $180^{\circ}$, the two orbits are co-planar and counter-rotating, which makes it easy to frequently form a limb-sounding (occultation) geometry and generate occultation events. Therefore, the number of 3-month occultation events reaches the maximum of about 5500 in this type of configuration. When the Rx RAAN and the Tx RAAN are equal, the $\mathrm{Rx}$ and Tx orbits are co-planar and running in the same direction, and the number of 3 -month occultation events is almost 0 .

To further explore the impact of the RAAN on the spatial-temporal distribution of occultation events, Figures 5 and 6 show the global coverage and the number trend beside the GCF trend of the occultation events for nine representative constellations, i.e., Rxraan30-Txraan30, Rxraan30-Txraan90, Rxraan30-Txraan150, Rxraan30-Txraan180, Rxraan30-Txraan210, Rxraan30-Txraan240, Rxraan30-Txraan270, Rxraan30-Txraan300, and Rxraan30-Txraan330.

As shown in Figure 5, fixing the Rx RAAN at $30^{\circ}$, when the Tx RAAN is $30^{\circ}$ (Figure 5a), the global coverage fraction of 3-month occultation events is only $3 \%$, and the occultation events are scattered across various latitude zones. With the increase in the Tx RAAN, the global coverage fraction first increases and then decreases. When the Tx RAAN is set to $210^{\circ}$ (Figure 5e), the global coverage fraction reaches the maximum $(97 \%)$, and when the Tx RAAN is set to $180^{\circ}$ (Figure $5 \mathrm{~d}$ ) and $240^{\circ}$ (Figure 5f), the global coverage fraction is about $91 \%$. The RAAN difference of the Rx and Tx is very important to the occultation global coverage and distribution. When it is close to $180^{\circ}$, global coverage of occultation events can form. For a RAAN difference of $60^{\circ}$ (Figure $5 b, i$ ) and $120^{\circ}$ (Figure $5 c, g$ ), the occultation events are mainly distributed in high latitude zones, and the GCF is low. When the difference is $90^{\circ}$ (Figure 5h), the occultation events are mainly distributed in the $-70^{\circ}$ 
to $-65^{\circ}$ latitude zone and the $65-70^{\circ}$ latitude zone, and the global coverage fraction is only $7 \%$.

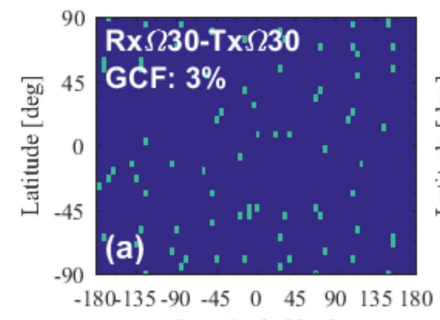

Longitude [deg]

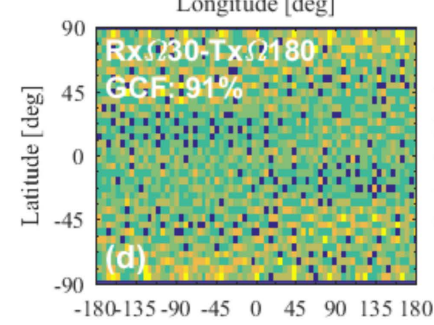

Longitude [deg]

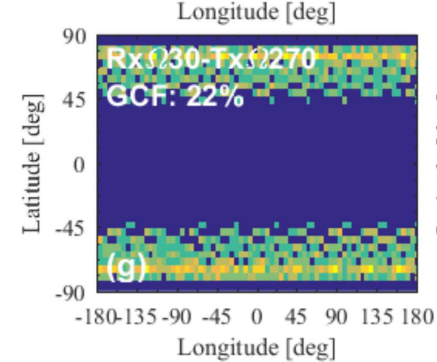

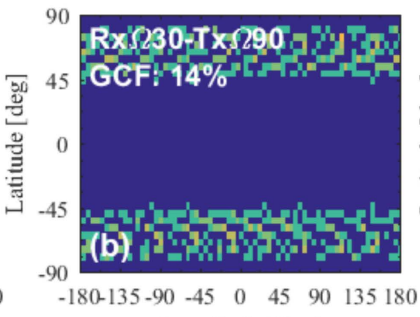

Longitude [deg]

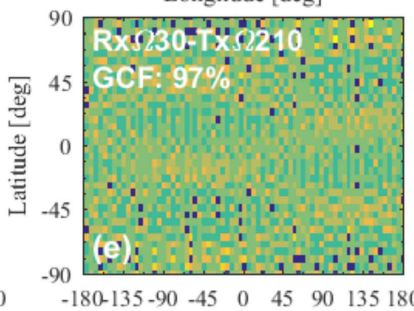

Longitude [deg]

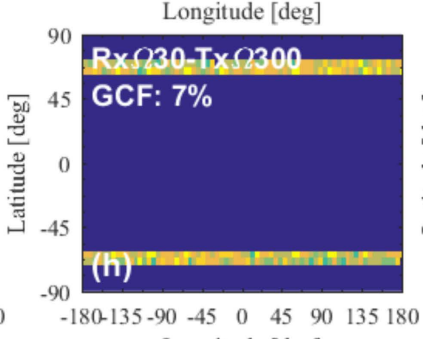

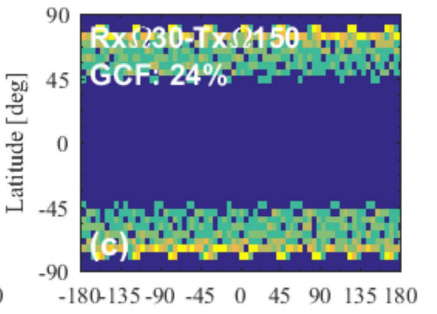

Longitude [deg]

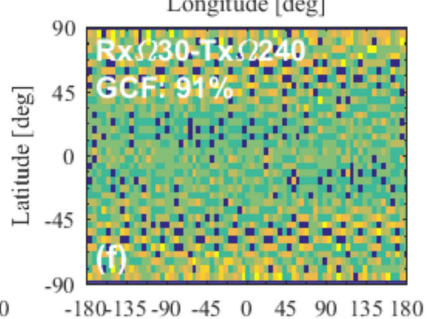

Longitude [deg]

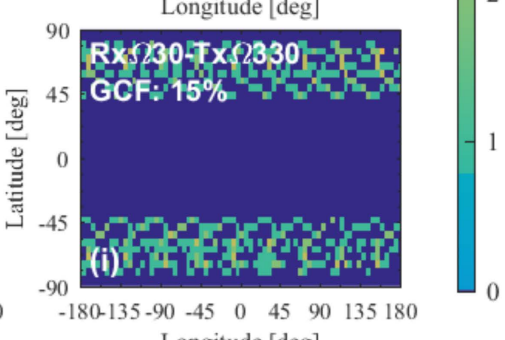

Figure 5. The global coverage of LEO-LEO occultation events for representative polar orbit constellations composed of a Rx satellite and a Tx satellite with particular orbital RAANs, illustrating the occultation numbers per $5^{\circ} \times 5^{\circ}$ latitude and longitude grid cell, while the GCFs were calculated using grid cell areas according to Equation (12).

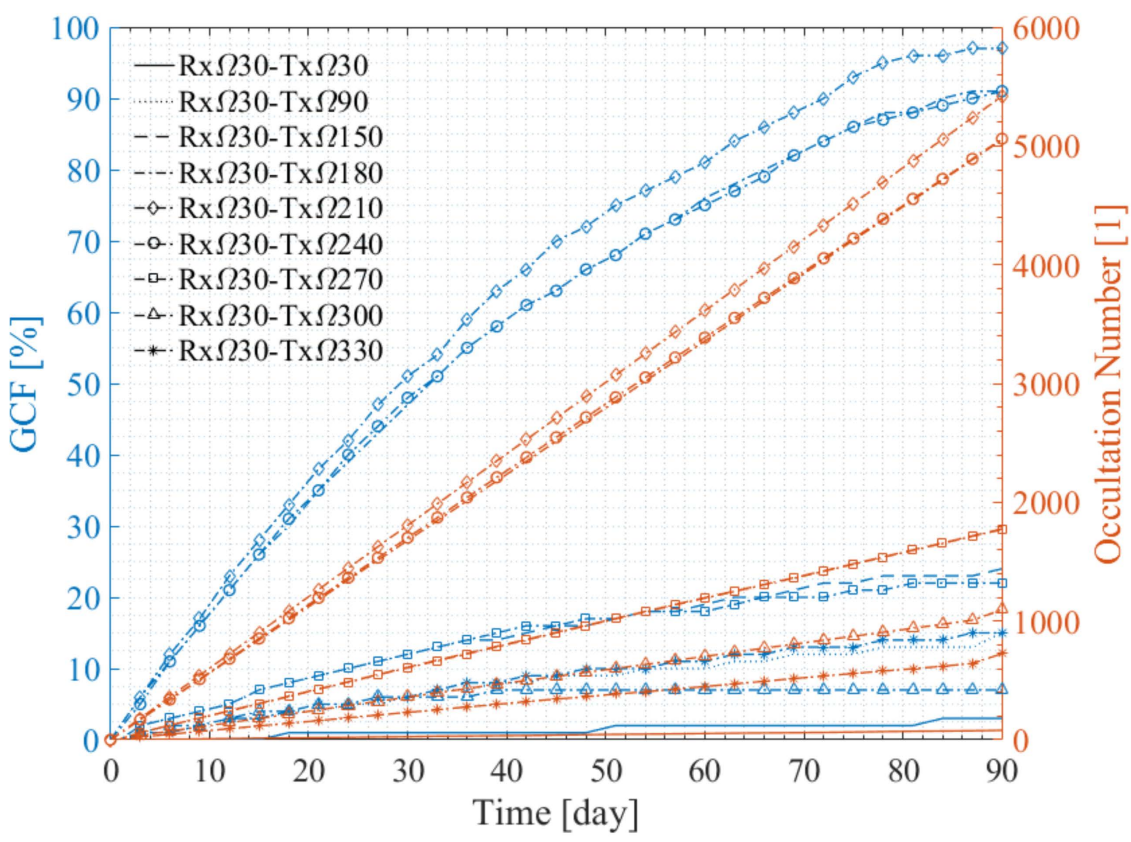

Figure 6. The accumulated time dependence of the LEO-LEO occultation event number (orange lines) and the global coverage fraction (GCF) (blue lines) for representative polar orbit constellations composed of a Rx satellite and a Tx satellite with particular orbital RAANs. 
Figure 6 illustrates the occultation number and the global coverage fraction trend with time for the constellations shown in Figure 5. One can see that the occultation number and global coverage fractions of these constellations increase almost linearly with time. When the RAAN difference of the Rx and Tx is $180^{\circ}$ (e.g., Rxraan30-Txraan210), the rate of growth in the occultation number is at its maximum (about 60 events per day), and the global coverage fraction first grows quickly and then tends to slow. When the RAAN difference of the Rx and Tx is $0^{\circ}$ (e.g., Rxraan30-Txraan30), the rate of growth in the occultation number is less than one event per day, and the 3-month global coverage fraction is only $3 \%$. Moreover, with the increase in the RAAN difference of the Rx and Tx from $0^{\circ}$ to $180^{\circ}$, the number of occultation events increases gradually from about 1 event to 60 events per day.

\subsubsection{Impact of the Height}

For the polar orbit constellations, one can see from Figure $2 \mathrm{c}$ that the number of occultation events that accumulated over 3 months gradually varies with the Rx and Tx height from about 4500 to 5500 events. The basic rule is that the lower the orbit is, and hence the shorter the orbital period, the more quickly the occultation number increases, since more orbit revolutions occur per day.

\subsection{Impact of the RAAN Precession on the LEO-LEO Occultation Events \\ 4.2.1. RAAN Precession Impact Analysis}

If the RAAN precession velocities of the Rx and Tx are different, the RAAN difference of the Rx and Tx will change with time, and the RAAN difference is a sensitive parameter for the LEO-LEO occultation event number and distribution. As shown in Figure 4, for the constellations with supplementary Rx and Tx inclinations, the number and global coverage fraction increase nonlinearly with time, and the occultation number grows very slowly during certain periods.

To explore the reason for the nonlinear trend of the occultation number and coverage fraction curves, Figure 7 illustrates the RAAN precession phenomenon of the Rxi100, Txi100, Rxi80, Rxi140, and Txi40 satellite orbits within the three months. The thick solid lines show the absolute RAAN differences of the Rx and Tx inclinations of the Rxi100Txi100, Rxi80-Txi100, and Rxi140-Txi40 constellations. One can see that when the Rx and Tx orbital inclinations are equal to each other (e.g., Rxi100-Txi100), their RAAN precession velocities are similar and the absolute RAAN difference remains around $180^{\circ}$. When the Rx and Tx orbital inclinations are supplementary (e.g., Rxi80-Txi100 and Rxi140-Txi40), their RAAN precession velocities are in opposite directions, and the absolute RAAN differences change periodically between $0^{\circ}$ and $180^{\circ}$, which is consistent with the RAAN precession law described by Formula (1).

When the absolute RAAN differences of the Rx and Tx are close to $180^{\circ}$, it is easy to form an occultation geometry and the total number of occultation events increases rapidly. When the absolute RAAN differences of the Rx and Tx are close to $0^{\circ}$, it is difficult to form an occultation geometry and the increase in the total number of occultation events stops. Comparing the relevant curves in Figures 4 and 7, the corresponding time periods are consistent with the abovementioned change trends of the occultation numbers and RAAN differences. This is also the reason why the NPO and MIO constellations with supplementary $\mathrm{Rx}$ and $\mathrm{Tx}$ inclinations have reduced occultation numbers.

However, for the NEO constellations, when the Rx and Tx inclinations are supplementary, the two orbits are counter-rotating and easily form the occultation geometry. The opposite direction of the RAAN precession accelerates the relative motion of the Rx and the Tx and helps to form the occultation geometry. Therefore, the NEO constellations with supplementary $\mathrm{Rx}$ and $\mathrm{Tx}$ inclinations have a large number of occultation events. For the NEO constellations with the same Rx and Tx inclinations, the Rx and Tx RAAN precession velocities are similar, and the RAAN difference remains around $180^{\circ}$ for a long time. This means that the Tx and Rx satellites are running in the same direction. In this setting, it is difficult to form an occultation geometric relationship. 


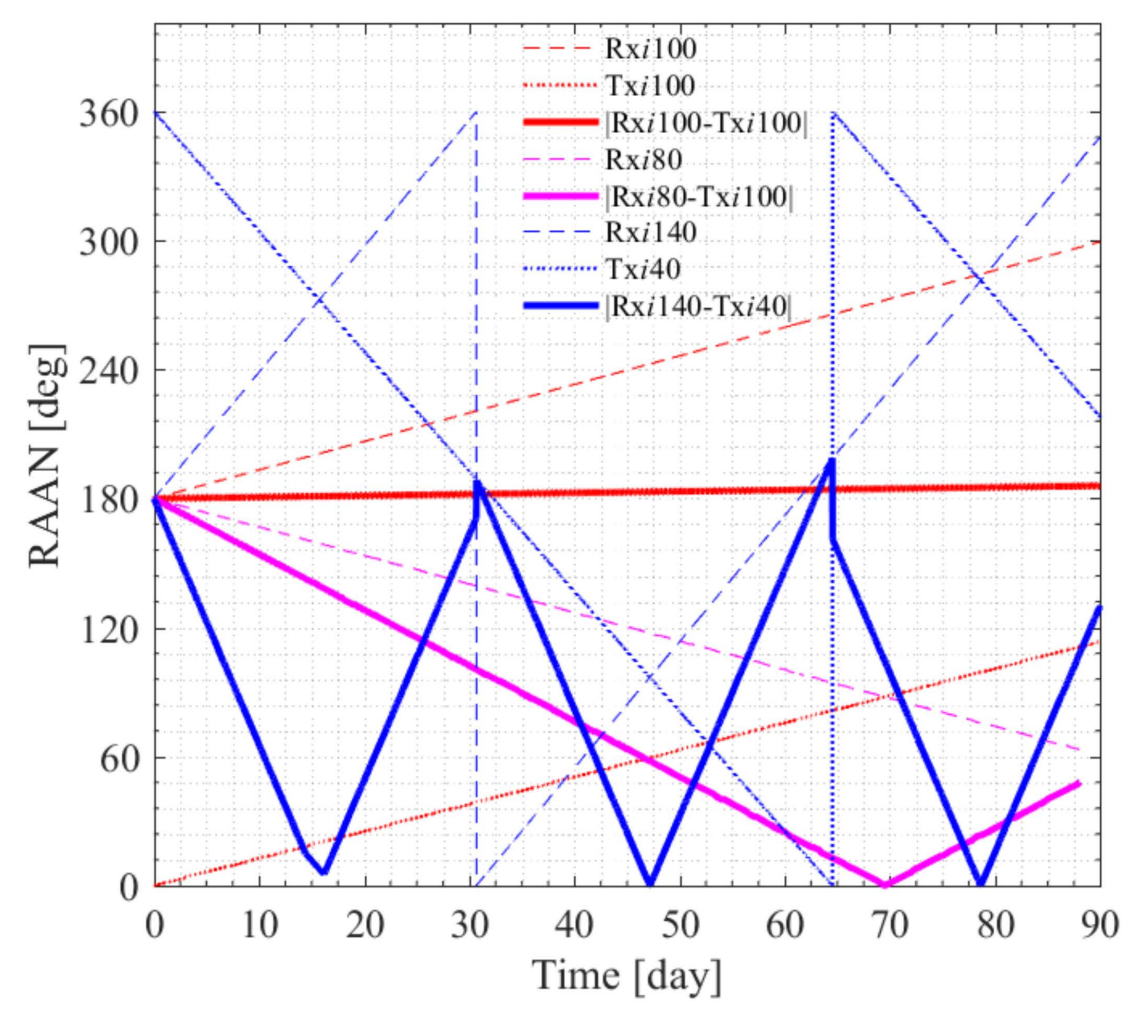

Figure 7. Rx and Tx RAANs and their absolute differences for Rxi100 (red dashed line), Txi100 (red dotted line), | Rxi100-Txi100 I (red bold line), Rxi80 (magenta dashed line), | Rxi80-Txi100 I (magenta bold line), Rxi140 (blue dashed line), Txi40 (blue dotted line), and I Rxi140-Txi40 I (blue bold line).

For the MIO constellation, when the Rx and Tx orbital inclinations are supplementary, the absolute RAAN differences change periodically between $0^{\circ}$ and $180^{\circ}$. The periods of the co-planar setting are short, so the occultation number is small. When the Rx and Tx orbital inclinations are equal, although the RAAN differences remain at $180^{\circ}$, the dihedral angle between the Rx and Tx orbital planes is large, which makes it difficult for the Rx and Tx satellites to see each other.

\subsubsection{Impacts of the Height on the Near-Polar Orbit Constellation Occultation Events}

Although the Tx and Rx orbital planes are not fully co-planar, it is still easy for the near-polar orbit (NPO) constellation with the same or very similar Rx and Tx inclinations to form the LEO-LEO occultation geometry and achieve global coverage, with a similar observation efficiency as a fully polar orbit constellation. Therefore, it is valuable to further investigate the NPO constellations. The impacts of the height on the constellation Rxi98Txi98, which is composed of two sun-synchronous orbits, and the constellation Rxi98-Txi82, which is composed of a sun-synchronous orbit and a supplementary orbit, were simulated using the variable separation approach. The main orbit parameters are shown in Table 2.

Figure 8 illustrates the number of 3-month occultation events of the constellations Rxi98-Txi98 and Rxi98-Txi82 as a function of orbital height. As shown in Figure 8a, the variation in the occultation number with the height of the Rxi98-Txi98 constellation is quite similar to that of the Rxi90-Txi90 constellation shown in Figure 2. The lower the orbit, the higher the number of occultation events. However, as shown in Figure 8b, the 3-month occultation number of the Rxi98-Txi82 constellation is smaller than those of the Rxi90-Txi90 and Rxi98-Txi98 constellations due to the effect of the opposite-direction RAAN precession of the Rx and Tx satellites. 
Table 2. The main orbital elements of the transmitting and receiving satellites for the near-polar orbit (NPO) constellations. The chosen set of values for some elements is denoted in the format of StartValue:Step:EndValue; simulations were performed using an ensemble of configurations stepping through all value settings for these elements.

\begin{tabular}{|c|c|c|}
\hline \multirow{2}{*}{$\begin{array}{l}\text { Orbit Elements } \\
\qquad(\mathrm{Rx} / \mathrm{Tx})\end{array}$} & \multicolumn{2}{|c|}{ Experimental Groups } \\
\hline & Rxi98-Txi98 & Rxi98-Txi82 \\
\hline I $\left(^{\circ}\right)$ & $98 / 98$ & $98 / 82$ \\
\hline $\operatorname{RAAN}\left({ }^{\circ}\right)$ & $180 / 0$ & $180 / 0$ \\
\hline H (km) & 400:100:1300/400:100:1300 & 400:100:1300/400:100:1300 \\
\hline $\mathbf{A P}\left({ }^{\circ}\right)$ & $80 / 80$ & $80 / 80$ \\
\hline Mo $\left({ }^{\circ}\right)$ & $210 / 30$ & $210 / 30$ \\
\hline E & $0.0001 / 0.0001$ & $0.0001 / 0.0001$ \\
\hline
\end{tabular}

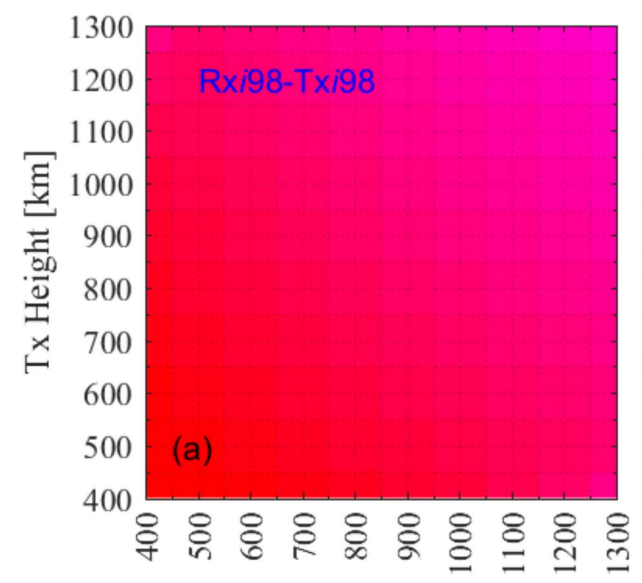

Rx Height $[\mathrm{km}]$

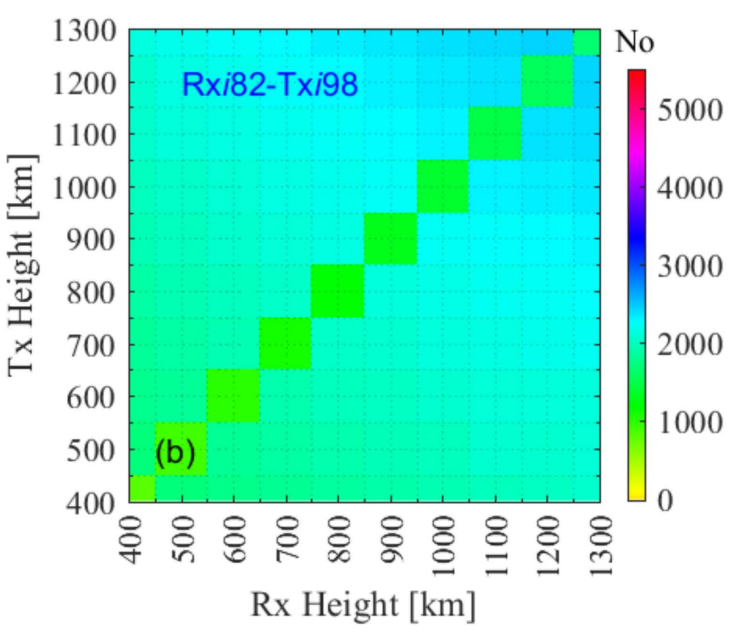

Figure 8. The variation in LEO-LEO occultation event numbers with the Rx and Tx orbital element of height for the Rxi98-Txi98 and Rxi82-Txi98 datasets on event accumulations over 3 months.

In the case of Rxi98-Txi82, the impact of height is obvious, especially when the Rx and Tx orbital heights are equal as the number of occultation events drops sharply. To explore how the number of occultation events varies with height, Figures 9 and 10 show the global coverage and the number trend beside the GCF trend of the occultation events for the Rxi98-Txi82 constellations with different height combinations.

As shown in Figures 9 and 10, when the Rx and Tx heights are equal, their velocities are the same, and the occultation events occur in the same latitude zone during each orbital period. Further, since the RAAN precession is in the opposite direction, the Rx and Tx satellites do not see each other for a long time. So, the occultation number is very small and the events are concentrated near the equator zone $(\mathrm{a}-\mathrm{c})$. As shown in Figure $9 \mathrm{~d}-\mathrm{f}$, fixing the Rx height at $400 \mathrm{~km}$, when the Tx height increases, the occultation number and the global coverage fraction increase. As shown in Figure 9g-i, fixing the Rx height at $1300 \mathrm{~km}$, when the Tx height increases, the occultation number and the global coverage fraction also increase. The occultation number and the global coverage fraction of the Rxh1300-Txh1200 constellation reach a maximum of approximately 2400 and $61 \%$, respectively. However, compared with the Rxi90-Txi90 and Rxi98-Txi98 constellations, the occultation number and the global coverage fraction of Rxi98-Txi82 are both smaller. 

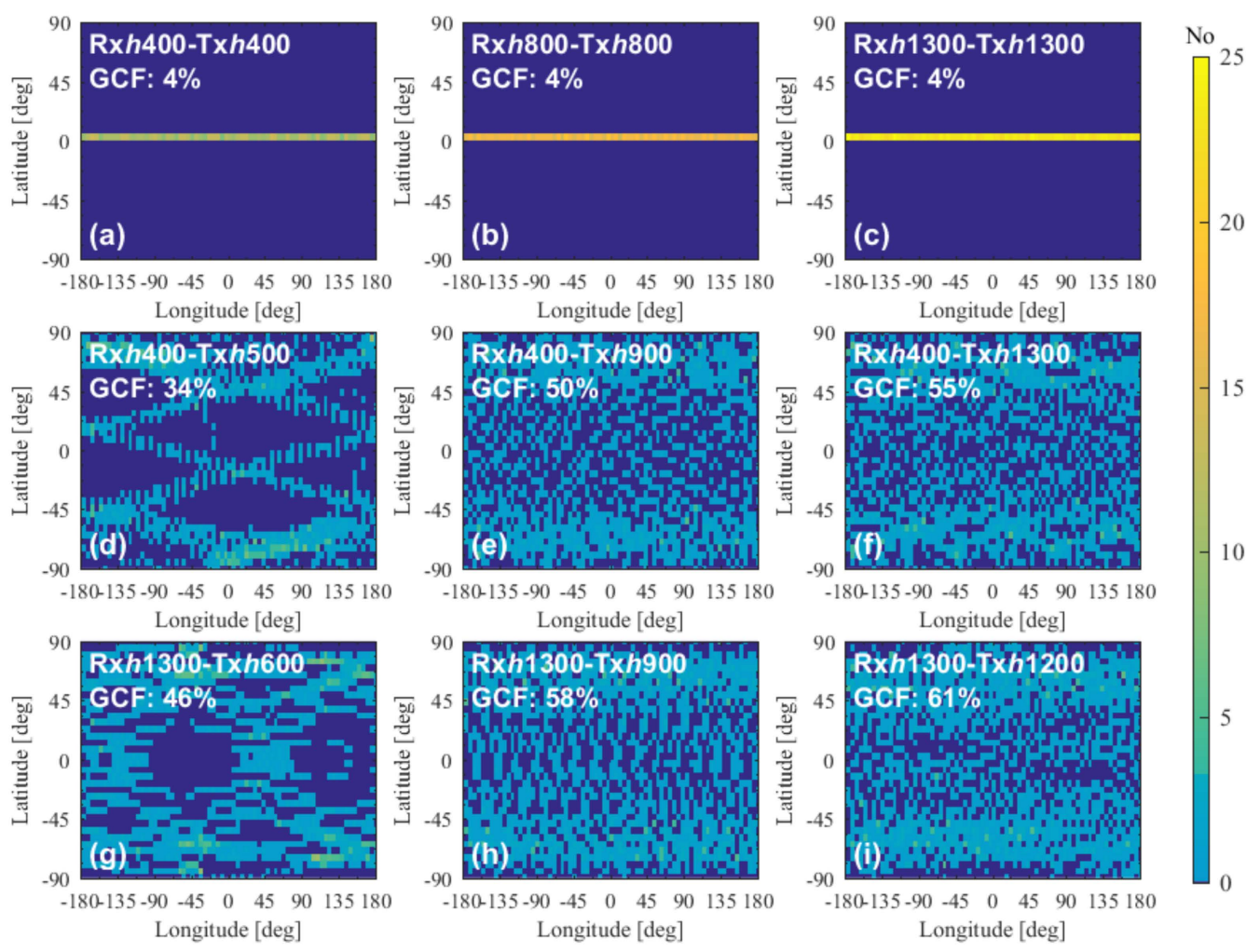

Figure 9. The global coverage of LEO-LEO occultation events for representative Rxi98-Txi82 constellations composed of a Rx satellite and a Tx satellite with particular orbital heights, illustrating the occultation numbers per $5^{\circ} \times 5^{\circ}$ latitude and longitude grid cell. The GCFs were calculated using grid cell areas according to Equation (12).

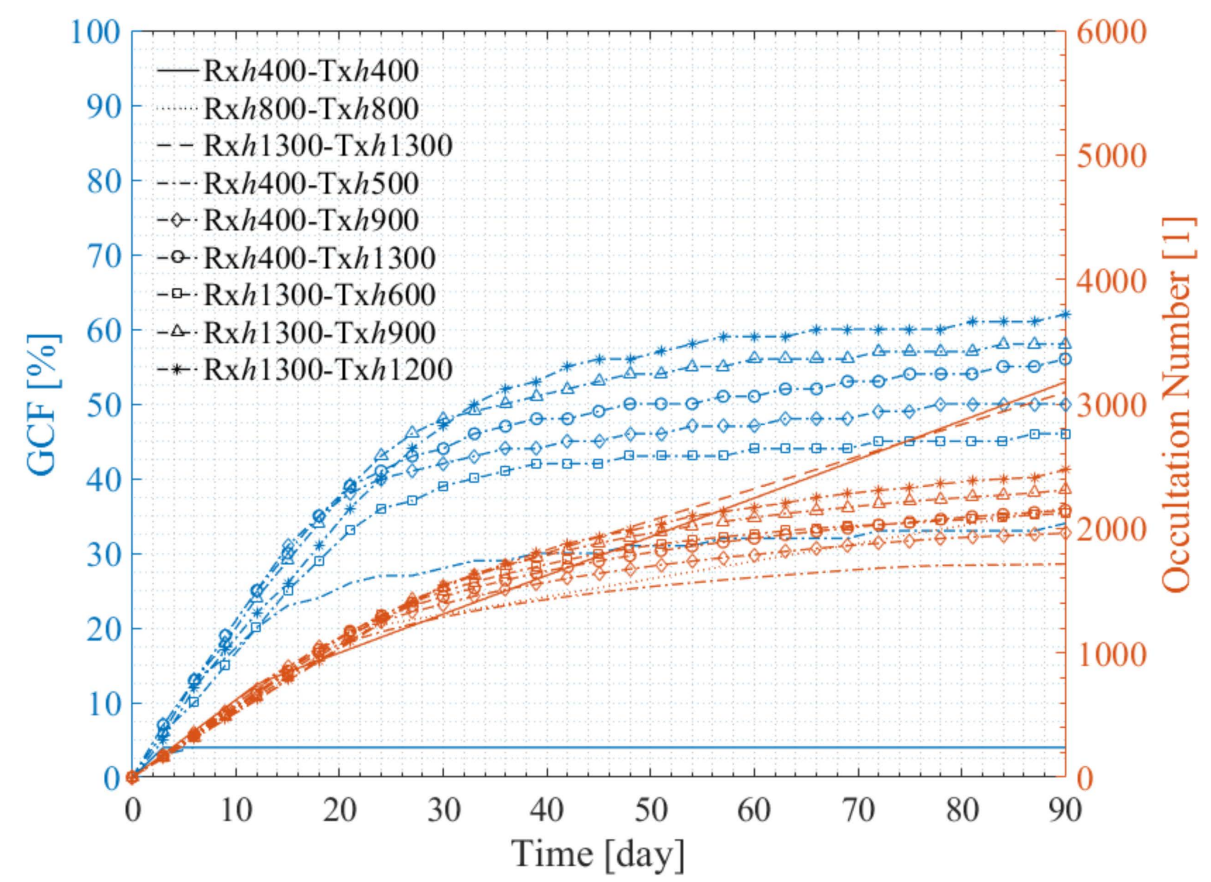

Figure 10. The accumulated time dependence of the LEO-LEO occultation event number (orange lines) and the global coverage fraction (GCF) (blue lines) for the representative Rxi98-Txi82 constellations composed of a Rx satellite and a Tx satellite with particular orbital heights. 


\subsection{Assessment of Multi-Rx and Multi-Tx LEO-LEO Occultation Constellation Performance 4.3.1. Orbital Parameter Setup for Multi-Satellite Experimental Constellations}

The analysis of impacts of orbital parameters on the LEO-LEO occultation performance showed that NPO constellations with the same Rx and Tx inclinations are good for observing the entire Earth with a great number of occultation events and global coverage. NEO constellations with supplementary $\mathrm{Rx}$ and Tx inclinations are good for observing middle and low latitude zones with a high number of occultation events and long revisiting times. Therefore, the observation efficiency of LEO-LEO occultation constellations was investigated by using these two types of orbits.

In this study, the two-orbit constellations are composed of one Rx orbit and one Tx orbit and have certain numbers of $R x$ and Tx satellites running in these prescribed orbits. Table 3 summarizes all cases using a concise notation. The types of constellations are denoted Rxi98-Txi98, Rxi140-Txi40, or something similar, with 2Rx/2Tx, 6Rx/6Tx, and 12Rx/12Tx specifying the numbers of satellites in these orbits. For example, Rxi98-Txi98 2Rx/2Tx denotes one such constellation in the first line of Table 3, where the choice of M0 for each of the two Tx/2 Rx satellites is also shown. Likewise, the multiple-orbit constellations are composed of certain numbers of Rxi98-Txi98 two-orbit constellations, e.g., Rxi98-Txi98 $2 \mathrm{Rx} / 2 \mathrm{Tx}$ orbit denotes two such orbit pairs (distinguished by their respective RAANs) as shown in the seventh line of Table 3.

Table 3. The main orbital parameters of the transmitting and receiving satellites for multi-Rx and multi-Tx constellations. The chosen set of values for some elements is denoted in the format of StartValue:Step:EndValue; simulations were performed using an ensemble of configurations stepping through all value settings for these elements.

\begin{tabular}{|c|c|c|c|c|}
\hline \multirow{2}{*}{\multicolumn{2}{|c|}{ Experiment Groups }} & \multicolumn{3}{|c|}{ Orbit Elements (Rx/Tx) } \\
\hline & & I $\left({ }^{\circ}\right)$ & RAAN ( $\left.{ }^{\circ}\right)$ & M0 ( $\left.{ }^{\circ}\right)$ \\
\hline \multirow{3}{*}{ Rxi98-Txi98 } & $2 \mathrm{Rx} / 2 \mathrm{Tx}$ & $98 / 98$ & $180 / 0$ & $30,120 / 150,240$ \\
\hline & $6 \mathrm{Rx} / 6 \mathrm{Tx}$ & $98 / 98$ & $180 / 0$ & $30: 60: 360 / 30: 60: 360$ \\
\hline & $12 \mathrm{Rx} / 12 \mathrm{Tx}$ & $98 / 98$ & $180 / 0$ & $30: 30: 360 / 30: 30: 360$ \\
\hline \multirow{3}{*}{ Rxi140-Txi40 } & $2 \mathrm{R} x / 2 \mathrm{Tx}$ & $140 / 40$ & $180 / 0$ & $30,120 / 150,240$ \\
\hline & $6 \mathrm{Rx} / 6 \mathrm{Tx}$ & $140 / 40$ & $180 / 0$ & $30: 60: 360 / 30: 60: 360$ \\
\hline & $12 \mathrm{Rx} / 12 \mathrm{Tx}$ & $140 / 40$ & $180 / 0$ & $30: 30: 360 / 30: 30: 360$ \\
\hline \multirow{3}{*}{$\begin{array}{l}\text { Multiple orbits of } \\
\text { Rxi98-Txi98 }\end{array}$} & 2Rx/2Txorbit & $98 / 98$ & $0,90 / 180,270$ & $30: 60: 360 / 30: 60: 360$ \\
\hline & 4Rx/4Txorbit & $98 / 98$ & 30:30:120/210:30:300 & $30: 60: 360 / 30: 60: 360$ \\
\hline & 6Rx/6Txorbit & $98 / 98$ & 0:30:150/180:30:330 & $30: 60: 360 / 30: 60: 360$ \\
\hline
\end{tabular}

For the specific orbital parameters, the orbital heights of the $\mathrm{Rx}$ and Tx satellites are fixed at $500 \mathrm{~km}$ and $600 \mathrm{~km}$, respectively, AP is fixed at $80^{\circ}$, and e is fixed at 0.0001 . Different LEO-LEO occultation constellations were designed by setting different values of the I, M0, and RAAN parameters. Six two-orbit and three multiple-orbit constellations were designed and evaluated, and their main orbital parameters are shown in Table 3.

In order to quantitatively analyze the observation efficiency of the multi-Rx and multiTx occultation constellations, the orbital parameters of the nine constellations shown in Table 3 were used to carry out a numerical experiment, in which 10-day occultation events were simulated, and the 1-day, 3-day, and 9-day global coverage fractions of occultation events were calculated.

\subsubsection{Performance of Two-Orbit Constellations}

Figure 11a-c, Figure 11d-f, and Figure 11g-i illustrate the 1-day, 3-day, and 9-day global coverage of occultation events for the Rxi98-Txi98 2Rx/2Tx, 6Rx/6Tx, and 12Rx/12Tx constellations, respectively. As shown in Figure 11a-c, the 1-day, 3-day, and 9-day global coverage fractions of $2 \mathrm{Rx} / 2 \mathrm{Tx}$ occultation events are about $8 \%, 25 \%$, and $63 \%$, respectively. As shown in Figure 11d-f, the 1-day, 3-day, and 9-day global coverage fractions of 6Rx/6Tx occultation events are about $50 \%, 76 \%$, and $100 \%$, respectively, which is much higher 
than those of the $2 \mathrm{Rx} / 2 \mathrm{Tx}$ constellation; specifically, the 9-day global coverage fraction reaches $100 \%$. As shown in Figure 11g-i, the 1-day and 3-day global coverage fractions of $12 \mathrm{Rx} / 12 \mathrm{Tx}$ occultation events reach $67 \%$ and $86 \%$, respectively. The 9 -day global coverage fraction of $12 \mathrm{Rx} / 12 \mathrm{Tx}$ occultation events reaches $100 \%$ with uniform global coverage, and the number of single-grid occultation event revisited times in some areas is more than 40 , which can basically meet the requirements of global climate monitoring [18].
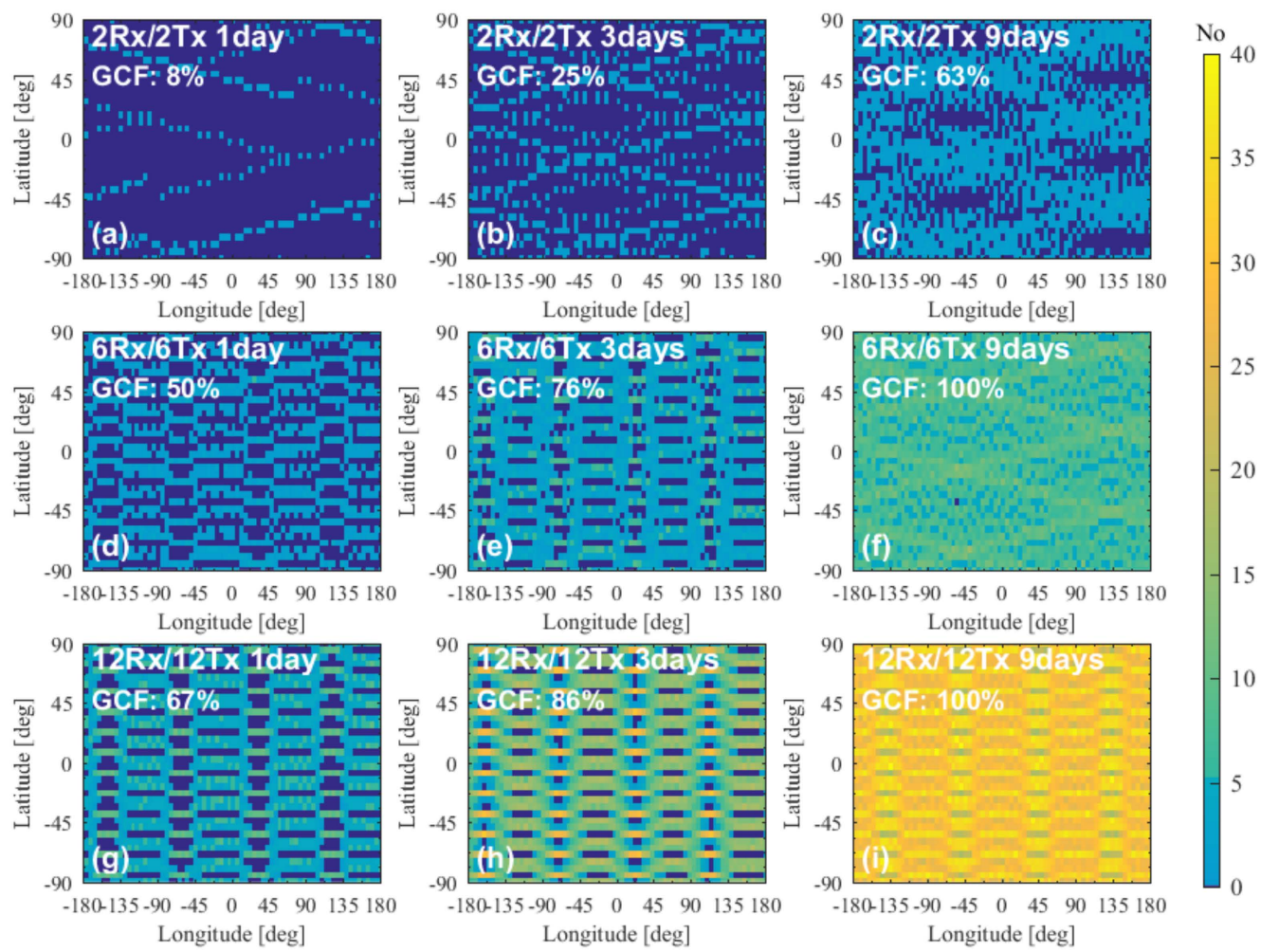

Figure 11. The global coverage of LEO-LEO occultation events for the $2 \mathrm{Rx} / 2 \mathrm{Tx}, 6 \mathrm{Rx} / 6 \mathrm{Tx}$ and $12 \mathrm{Rx} / 12 \mathrm{Tx}$ constellations with the Rxi98 and Txi98 inclination orbital planes, illustrating the occultation numbers per $5^{\circ} \times 5^{\circ}$ latitude and longitude grid cell. The GCFs were calculated using grid cell areas according to Equation (12).

The global coverage fractions and the number trend of the occultation events for the Rxi98-Txi98 2Rx/2Tx, 6Rx/6Tx, and 12Rx/12Tx constellations are shown in Figure 12. One can see that with the increase in the number of $\mathrm{Rx}$ and $\mathrm{Tx}$ satellites, the slope of the number and GCF curves increases significantly. Specifically, the 6Rx/6Tx constellation achieves a global coverage fraction of $100 \%$ in 8 days, and the 10-day occultation number reaches more than 61,000 . The $12 \mathrm{Rx} / 12 \mathrm{Tx}$ constellation achieves a global coverage fraction of $100 \%$ in just 7 days, and the 10-day occultation number reaches more than 86,000 .

Figure 13a-c, Figure 13d-f, and Figure 13g-i illustrate the 1-day, 3-day, and 9-day global coverage of occultation events for the Rxi140-Txi40 2Rx/2Tx, 6Rx/6Tx, and 12Rx/12Tx constellations, respectively. As shown in Figure 13a-c, the 1-day, 3-day, and 9-day global coverage fractions of $2 \mathrm{Rx} / 2 \mathrm{Tx}$ occultation events are about $11 \%, 32 \%$, and $49 \%$, respectively. As shown in Figure 13d-f, the 1-day, 3-day, and 9-day global coverage fractions of 6Rx/6Tx occultation events are about $50 \%, 70 \%$, and $75 \%$, respectively, which is much higher than those of the $2 \mathrm{Rx} / 2 \mathrm{Tx}$ constellation, and the number of times a single grid cell is revisited has increased significantly. As shown in Figure 13g-i, the 1-day, 3-day, and 9-day global coverage fractions of $12 \mathrm{Rx} / 12 \mathrm{Tx}$ occultation events reach $69 \%, 71 \%$, and $77 \%$, respectively. For the 9-day global coverage fraction of $12 \mathrm{Rx} / 12 \mathrm{Tx}$ occultation events, the number of times a single grid is revisited in some areas is more than 70 , which is beneficial to the observation of low and middle latitude regions, but cannot $100 \%$ cover the Earth. 


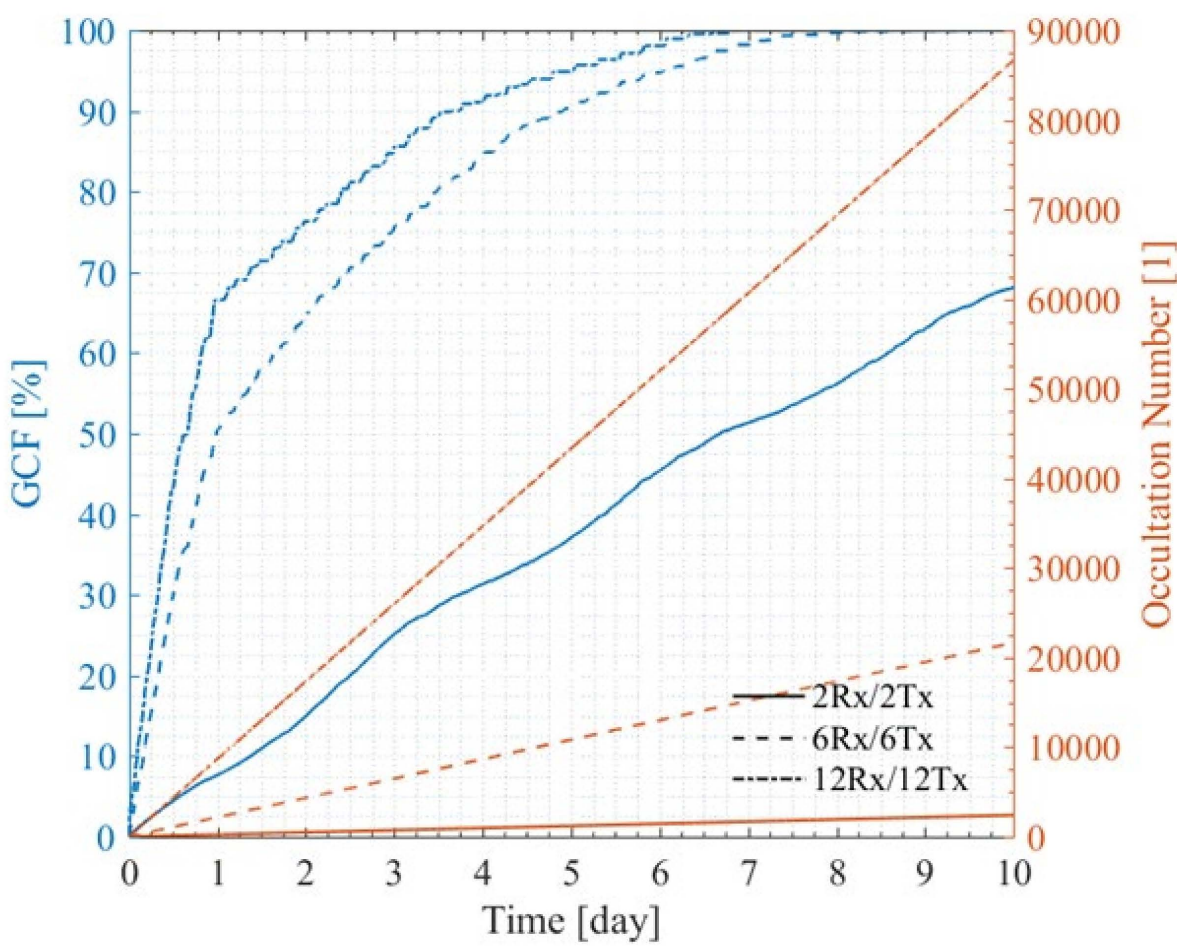

Figure 12. Accumulated time dependence of the global coverage fraction (GCF) (blue lines) and the occultation event number (orange lines) for the $2 \mathrm{Rx} / 2 \mathrm{Tx}, 6 \mathrm{Rx} / 6 \mathrm{Tx}$, and $12 \mathrm{Rx} / 12 \mathrm{Tx}$ constellations with the Rxi98 and Txi98 inclination orbital planes.
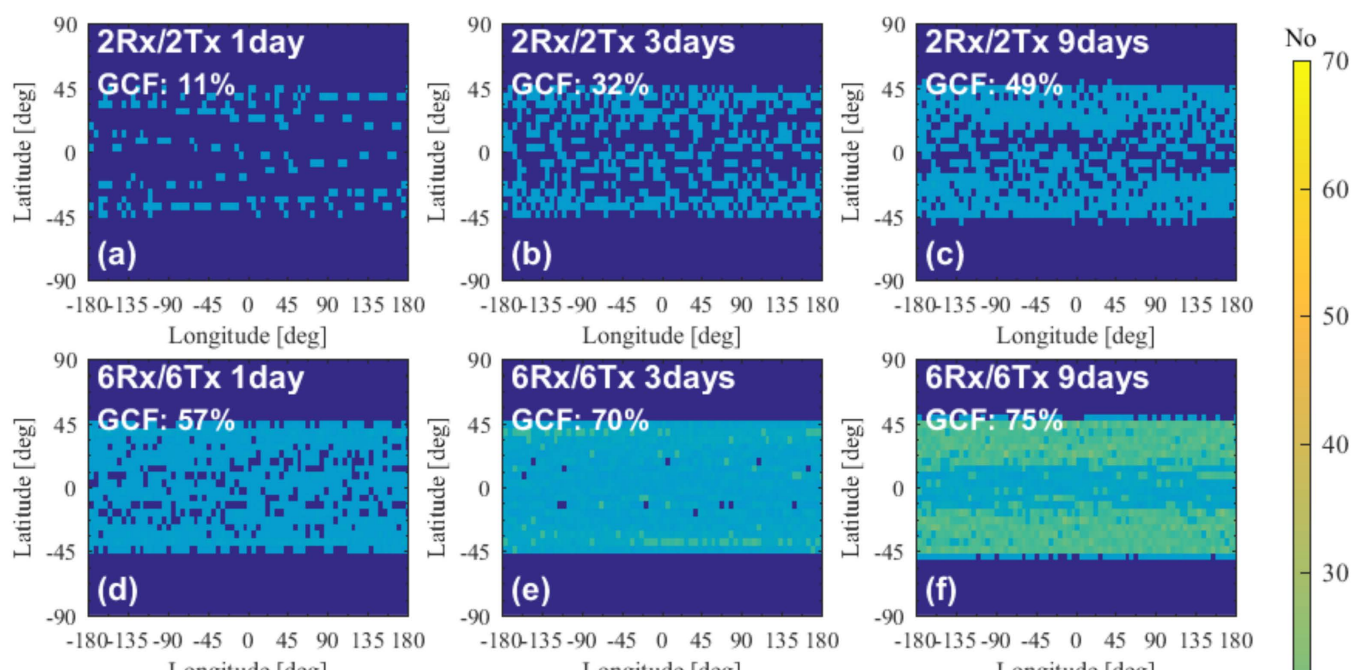

50
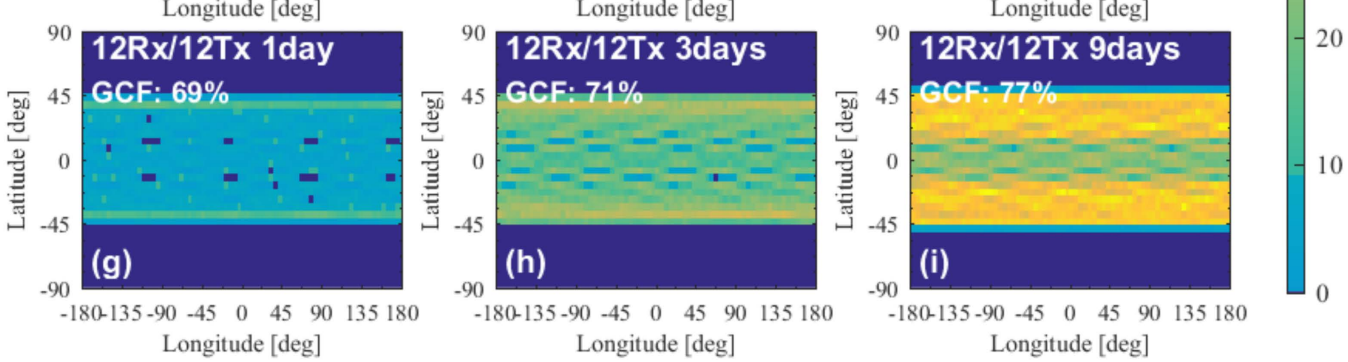

Figure 13. The global coverage of LEO-LEO occultation events for the $2 R x / 2 T x, 6 R x / 6 T x$, and $12 \mathrm{Rx} / 12 \mathrm{Tx}$ constellations with the Rxi140 and Txi40 inclination orbital planes, illustrating the occultation numbers per $5^{\circ} \times 5^{\circ}$ latitude and longitude grid cell. The GCFs were calculated using grid cell areas according to Equation (12). 
The global coverage fraction and the number trend of the occultation events for the Rxi140-Txi40 2Rx/2Tx, 6Rx/6Tx, and 12Rx/12Tx constellations are shown in Figure 14. One can see that with the increase in the number of Rx and Tx satellites, the slope of the number curves increases significantly. However, the GCF increases rapidly at first and then remains at a certain level, at which point essentially no more new grid cells start to be covered and, hence, the GCF saturates (and only the number of events in cells already covered increases). Although the number of 10 -day $12 \mathrm{Rx} / 12 \mathrm{Tx}$ occultation events reaches more than 61,000 , the maximum GCF is $77 \%$. The 10 -day occultation number of the $6 \mathrm{Rx} / 6 \mathrm{Tx}$ constellation is about 15,000 , and the GCF is about $75 \%$. These results indicate that, due to the lower inclinations, the NEO constellations cannot observe the high latitude region.

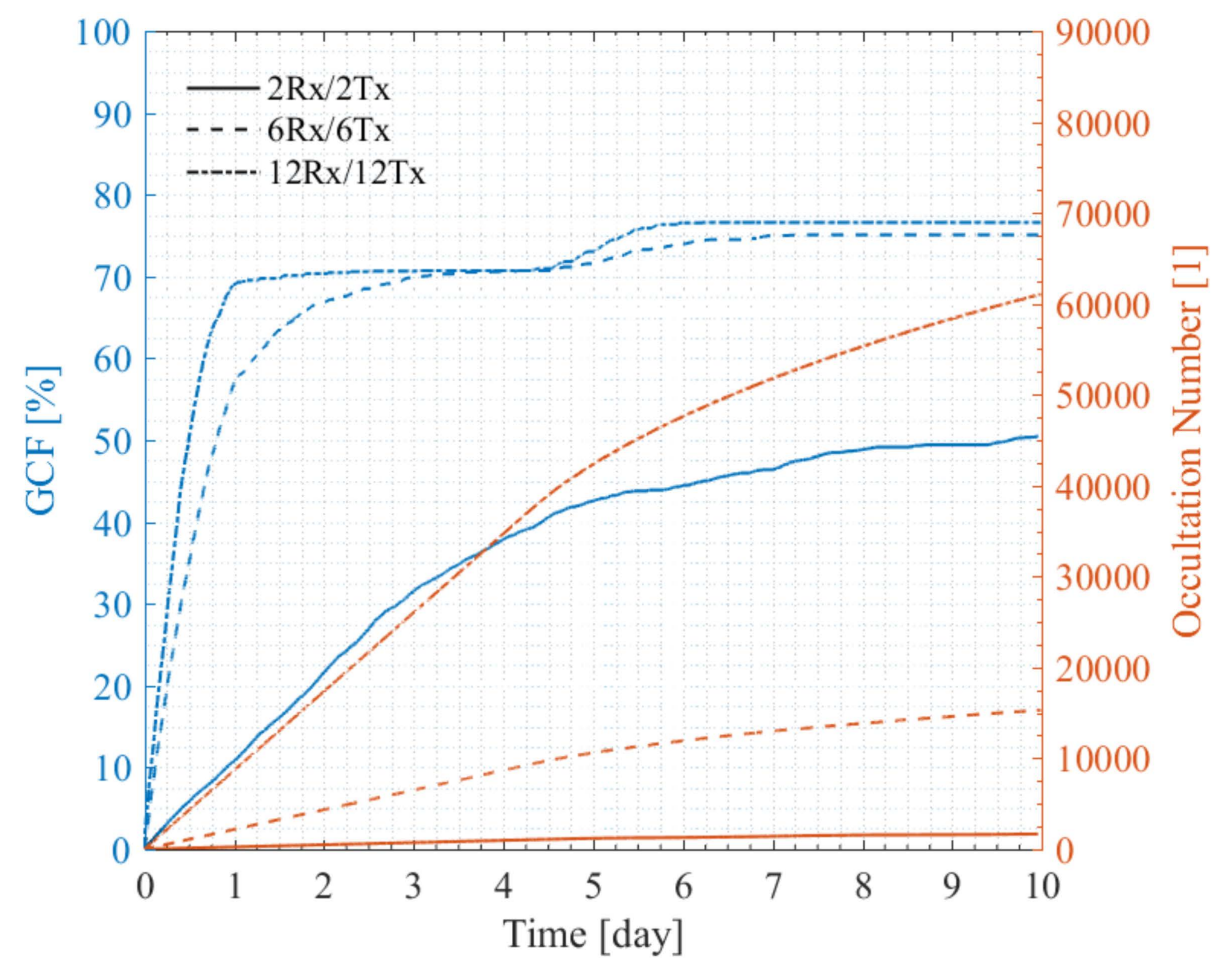

Figure 14. Accumulated time dependence of the global coverage fraction (GCF) (blue lines) and the occultation event number (orange lines) for the $2 \mathrm{Rx} / 2 \mathrm{Tx}, 6 \mathrm{Rx} / 6 \mathrm{Tx}$, and $12 \mathrm{Rx} / 12 \mathrm{Tx}$ constellations with the Rxi140 and Txi40 inclination orbital planes.

\subsubsection{Performance of Multiple-Orbit Constellations}

Figure 15a-c, Figure 15d-f, and Figure 15g-i illustrate the 1-day, 3-day, and 9-day global coverage of occultation events for the $2 \mathrm{Rx} / 2 \mathrm{~T} x$ orbit, $4 \mathrm{Rx} / 4 \mathrm{Tx}$ orbit, and 6Rx/6Txorbit constellations, respectively. As shown in Figure 15a-c, the global coverage fraction of 1-day $2 \mathrm{Rx} / 2$ Txorbit occultation events reaches $98 \%$ with uniform global coverage. The 3-day global coverage and the 9-day global coverage of $2 \mathrm{Rx} / 2 \mathrm{Txorbit}$ occultation events reach $100 \%$, and the number of single-grid 9-day occultation event revisited times in some areas is more than 50. Compared with the two-orbit 12Rx/12Tx constellation in Figure 11g-i, setting 24 satellites on four orbits, as in the $2 \mathrm{Rx} / 2$ Txorbit configurations, makes it easier to form 100\% global coverage. As shown in Figure 15d-i, the 4Rx/4Txorbit and 6Rx/6Txorbit constellations achieve $100 \%$ global coverage just in one day, and the number of times the 9-day $6 \mathrm{Rx} / 6 \mathrm{Tx}$ orbit occultation events are revisited in a single grid in most areas is more than 200. 


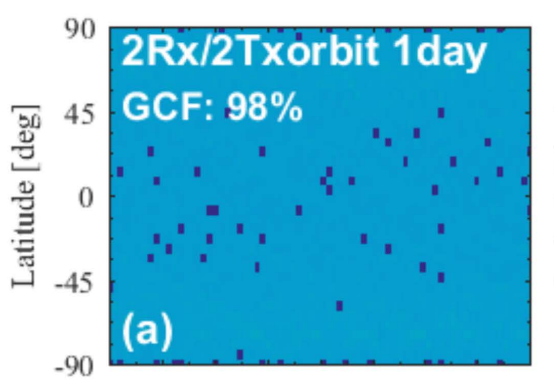

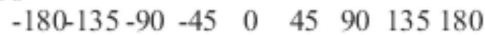
Longitude [deg]
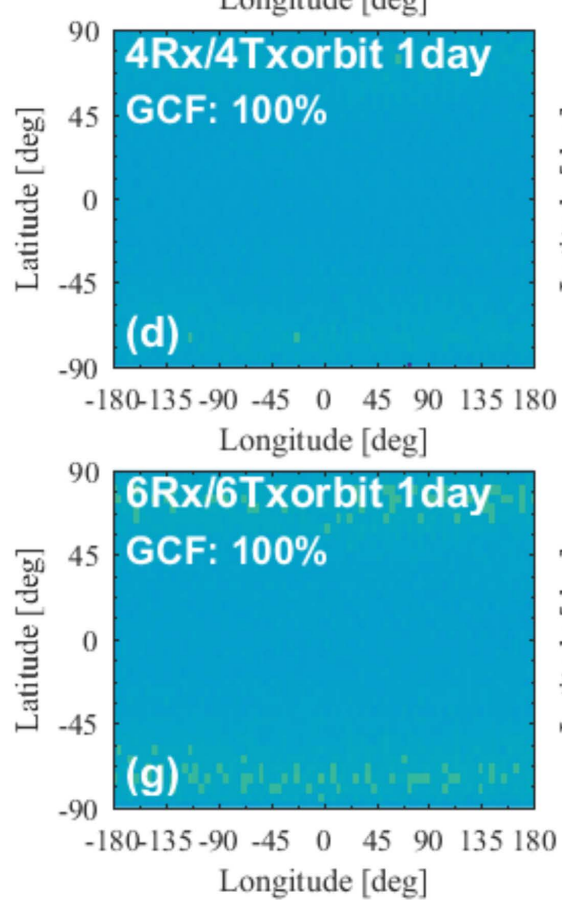

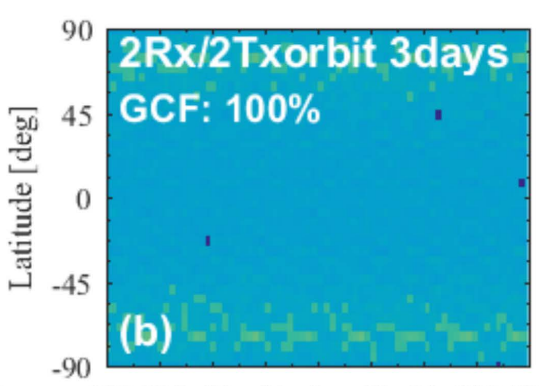

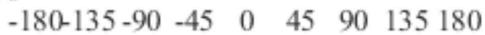
Longitude [deg]

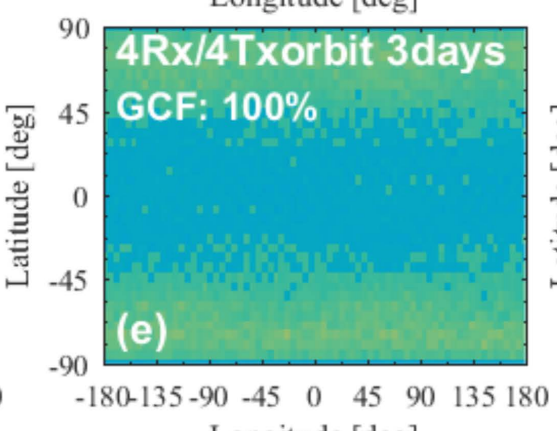

Longitude [deg]

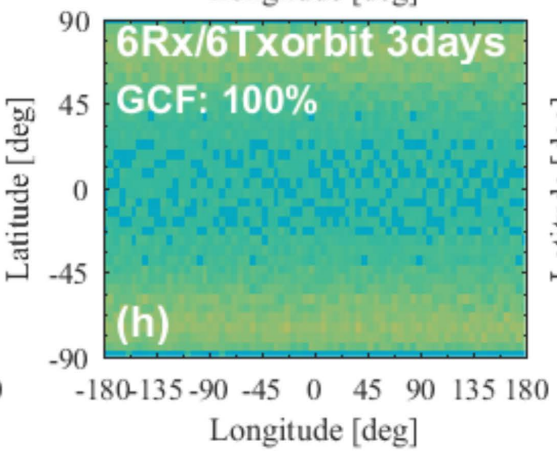

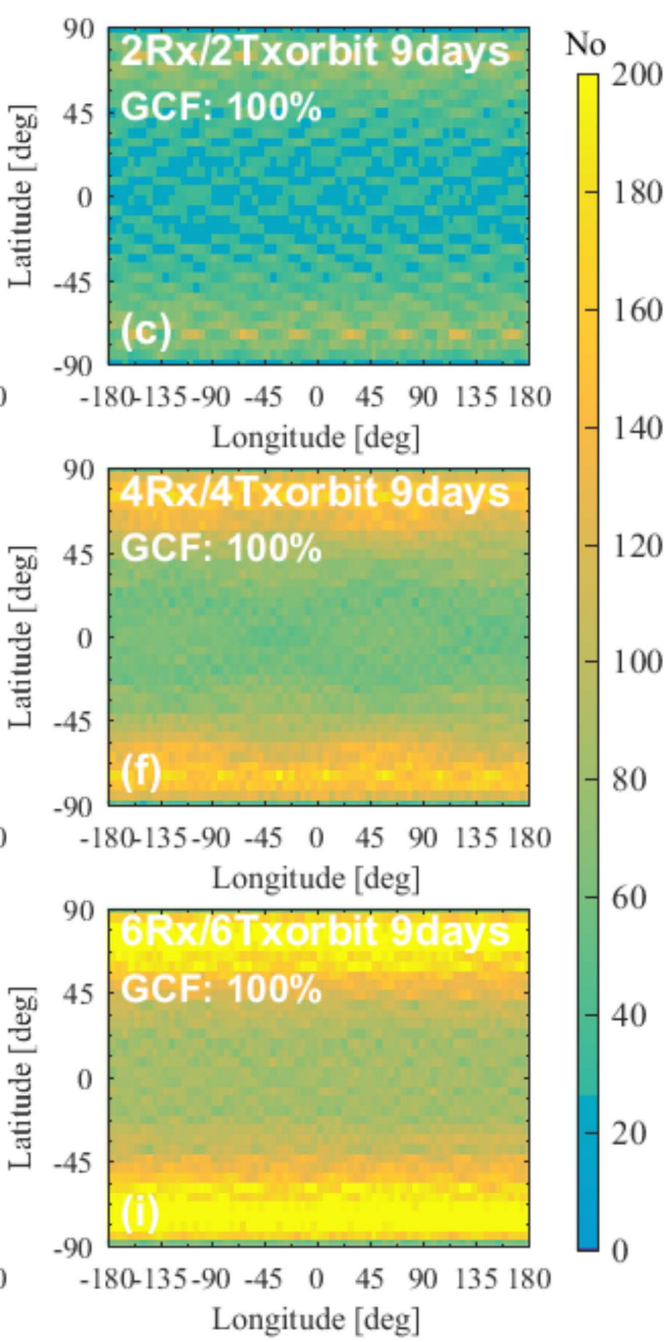

Longitude [deg]

Figure 15. The global coverage of LEO-LEO occultation events for the $2 \mathrm{Rx} / 2 \mathrm{Tx}$ orbit, $4 \mathrm{Rx} / 4 \mathrm{Txorbit}$, and $6 \mathrm{Rx} / 6 \mathrm{Txorbit}$ constellations with the Rxi98 and Txi98 inclination orbital planes, illustrating the occultation numbers per $5^{\circ} \times 5^{\circ}$ latitude and longitude grid cell. The GCFs were calculated using grid cell areas according to Equation (12).

The global coverage fraction and the number trend of the occultation events for the 2Rx/2Txorbit, 4Rx/4Txorbit, and 6Rx/6Txorbit constellations are shown in Figure 16. One can see that with the increase in the number of orbits, the slope of the number curves increases significantly; specifically, the rates of growth in the number of $4 \mathrm{Rx} / 4 \mathrm{Tx}$ xorbit and 6Rx/6Txorbit occultation events are more than 22,500 times/day and 38,000 times/day, respectively. The $4 \mathrm{Rx} / 4 \mathrm{Tx}$ orbit and $6 \mathrm{Rx} / 6 \mathrm{Tx}$ orbit constellations achieve $100 \%$ global coverage just in $18 \mathrm{~h}$ and $12 \mathrm{~h}$, respectively. It can be seen that the LEO-LEO occultation constellation composed of 36-72 satellites can meet the basic requirements of global numerical weather prediction in terms of occultation numbers and timeliness. 


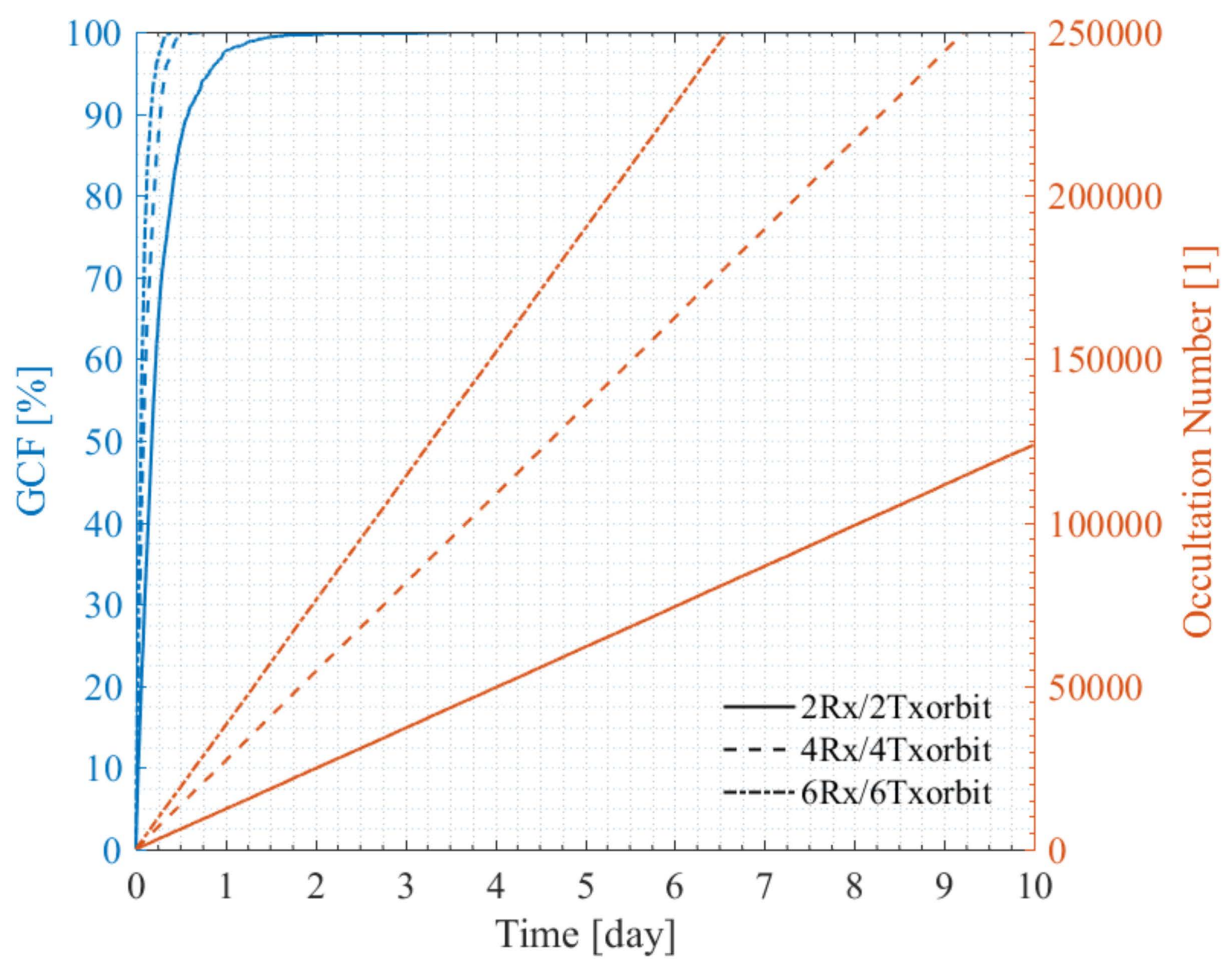

Figure 16. Accumulated time dependence of the global coverage fraction (GCF) (blue lines) and the occultation event number (orange lines) for the $2 \mathrm{Rx} / 2 \mathrm{Tx}$ orbit, $4 \mathrm{Rx} / 4 \mathrm{Tx}$ orbit and $6 \mathrm{Rx} / 6 \mathrm{Tx}$ orbit constellations with the Rxi98 and Txi98 inclination orbital planes.

\section{Discussions}

LEO-LEO occultation is an advanced and supplementary remote sensing technique for GNSS RO, but there is currently no space-borne LEO-LEO occultation constellation. Nowadays, with the development of small-satellite and instrument miniaturization technologies as well as the demonstration of the LEO-LEO occultation technique by numerical and ground-based experiments, there are more opportunities for the demonstration of large, space-borne, and operational LEO-LEO constellations. Therefore, it is the right time to conduct a detailed LEO-LEO occultation orbit analysis and a constellation assessment to provide references for future LEO-LEO occultation constellation designs.

\subsection{Summary of the Impacts of the Orbital Parameters on the LEO-LEO Occultation Events}

The impacts of the orbital parameters of inclination, RAAN, height, and AP on the number and global coverage of LEO-LEO occultation events were investigated using the variable separation approach based on a pair of Rx and Tx satellites. The numerical experiment results reveal that: (1) the inclination and RAAN are the main impact parameters followed by orbital height, while the impact of AP is small; (2) co-planar and counterrotating Rx and Tx satellites constitute an ideal two-satellite LEO-LEO constellation to form the occultation geometry easily; (3) for the polar orbit constellation, when the RAAN difference of the $\mathrm{Rx}$ and $\mathrm{Tx}$ is $180^{\circ}$, meaning that the orbits are co-planar and counter-rotating, it is easy to form the occultation geometry; (4) the polar orbit and NPO constellations easily achieve global coverage of occultation events; and (5) for the NEO constellation, when the Rx and Tx inclinations are supplementary, it is easy to form the occultation geometry, and the occultation events are mainly distributed in the low and middle latitude zones.

It should be noted that the results on the inclination, RAAN, and height shown in Figure 2 were obtained for polar orbit constellations without the effect of the RAAN precession, while the results for the inclination experimental group were affected by the inclination and RAAN precession. The inclination and RAAN define the relative position and running direction of the $\mathrm{Rx}$ and Tx satellites and are key parameters of LEO-LEO 
occultation orbital design, so it is necessary to carefully design the satellite orbit's inclination and RAAN to obtain more LEO-LEO occultation events and a better distribution.

The RAAN precession is an important orbital perturbation that cannot be neglected in the LEO-LEO occultation orbital and constellation design. The RAAN precession affects the number and distribution of LEO-LEO occultation events by changing the Rx and Tx RAANs. For particular constellations, the RAAN precession period may cause the repetition of the behavior and characteristics of the LEO-LEO occultation events as shown in Figure 4.

\subsection{Summary of the Performances of the Multi-Rx and Multi-Tx Constellations}

The assessment of the performances of the multi-satellite constellations showed that: (1) the number of LEO-LEO occultation events increases rapidly with the increase in the number of satellites and orbits; (2) with the NPO constellation, it is easy to achieve global coverage, and the NEO constellation is beneficial for middle and low latitude zonal observation; (3) for the unconstrained LEO-LEO occultation constellation design, it is advisable to adopt a polar orbit or at least NPO constellations; (4) to co-utilize existing or planned satellites to achieve the demonstration of the LEO-LEO technique by such platforms, NEO constellations can also be used; (5) to obtain more LEO-LEO occultation events and rapid global coverage, the two-orbit constellation is preferred for less than 24 satellites; otherwise, the multi-orbit constellation is recommended; and (6) a welldesigned LEO-LEO occultation constellation composed of 36-72 satellites can meet the basic requirements of global numerical weather prediction in terms of occultation event numbers and timeliness.

\subsection{Outlook on the Potential for LEO-LEO Occultation Demonstration and Operational Constellations}

The development and establishment of spacecraft such as space stations and meteorological satellites provide experimental platforms for potential LEO-LEO occultation demonstration opportunities. With such an orbit-constrained spacecraft, only (at least) one additional satellite needs to be launched to construct a pair of Rx and Tx LEO-LEO demonstration satellites. Taking a space station as an example, typically its orbit inclination is around $45^{\circ}$ and its height is about $400 \mathrm{~km}$, which means that it can be used as an occultation signal transmitting (Tx) platform. Thus, only a small receiving (Rx) satellite running in a retrograde orbit, with an inclination of about $140^{\circ}$ and a height of about $600 \mathrm{~km}$, is needed to construct a LEO-LEO constellation similar to the Rxi140-Txi40 one shown in Figures 3 and 4. The distribution of its LEO-LEO occultation events is in this case similar to that shown in Figure 3h, while the occultation number and GCF accumulated time dependence are similar to the triangle lines shown in Figure 4, which show about 3000 occultation events in 90 days and a 63\% Earth coverage fraction.

On the other hand, with the development of LEO satellite constellations with large numbers of satellites, such as the Iridium, Spire, and Starlink constellations, the nextgeneration space-based LEO constellations will tend to integrate satellite communication, satellite navigation, and satellite remote sensing functions in one system. Such a LEO constellation concept has also been proposed in China. If this kind of LEO constellation can be well designed as described in Section 4.3, it will yield a large amount of LEOLEO occultation data, which can be used for global numerical weather prediction and climate monitoring.

\section{Conclusions}

From the results and discussion we can draw the following conclusions: (1) the inclination and RAAN are the main orbital parameters impacting the occultation event number and coverage, followed by the orbital height; in particular, the RAAN precession is an important orbital perturbation that cannot be neglected in the LEO-LEO occultation constellation design; (2) co-planar and counter-rotating Rx and Tx orbits were confirmed to provide an ideal two-satellite LEO-LEO constellation; (3) polar orbit and near-polar orbit 
(NPO) constellations make it easy to achieve global coverage of occultation events, and hence are the best choices for an unconstrained LEO-LEO occultation constellation design; (4) to co-utilize existing or planned satellites as platforms to achieve the demonstration of the LEO-LEO technique, the near-equatorial orbit (NEO) constellation can also be used; and (5) the number of LEO-LEO occultation events increases rapidly with the increase in the number of satellites and orbits; constellations with 48 and 72 satellites deliver more than 22,500 and 38,000 occultation events per day, respectively, and can achieve $100 \%$ global coverage within $18 \mathrm{~h}$ and $12 \mathrm{~h}$, respectively. The spatio-temporal coverage of occultation events can in this way fully achieve global numerical weather prediction and climate monitoring requirements.

In this paper, our work mainly focused on the impacts of orbital parameters of the $\mathrm{Rx}$ and Tx satellites on the LEO-LEO occultation number and global coverage fraction. Regarding the LEO-LEO constellation design and quantitative analysis, we took six two-orbit and three multiple-orbit constellations as representative examples to assess the performance of LEO-LEO occultation constellations with different satellite numbers and orbital planes. In our future work, the impacts of orbital parameters on the LEO-LEO occultation geometry and then on the atmospheric products, as well as the constellation optimization approaches, will be further investigated in more detail.

Author Contributions: Conceptualization, C.L., G.K., Y.S. and X.W.; methodology, C.L., G.K., V.P., L.T., Q.D. and W.B.; software, C.L., P.H. and G.T.; validation, C.L., C.W. and L.T.; formal analysis, C.L.; investigation, C.L., G.K. and L.T.; resources, Y.S., G.K. and X.W.; writing-original draft preparation, C.L.; writing—review and editing, G.K.; reviewing V.P.; visualization, C.L. and G.K.; supervision, G.K. and Y.S.; project administration, C.L., X.W. and Y.S. All authors have read and agreed to the published version of the manuscript.

Funding: This research was partially funded by the National Natural Science Foundation of China (grant nos. 41775034, 41405039, and 41606206), the Youth Innovation Promotion Association of the Chinese Academy of Sciences (grant no. 2019151), the Strategic Priority Research Program of the Chinese Academy of Sciences (grant no. XDA15021002), the Youth Talents Program Foundation of the Beijing Organization Department (grant no. 2018000097607G380), and the FengYun-3 (FY-3) Global Navigation Satellite System Occultation Sounder (GNOS) development and manufacture project led by NSSC, CAS. The research at the WEGC/University of Graz was partially supported by the Austrian Research Promotion Agency (FFG) project ATROMSAF2 (ASAP-15 project no. 873696) based on Austrian Space Applications Programme (ASAP) funds provided by the Ministry for Climate, Environment, Energy, Mobility, Innovation, and Technology (BMK).

Acknowledgments: The authors thank Marc Schwaerz (WEGC/University of Graz, Austria) for the valuable support with the development of the EGOPS software that was relevant to this study.

Conflicts of Interest: The authors declare no conflict of interest.

\section{References}

1. Melbourne, W.G.; Davis, E.S.; Duncan, C.B.; Hajj, G.A.; Hardy, K.R.; Kursinski, E.R.; Meehan, T.K.; Yong, L.E.; Yunck, T.P. The application of spaceborne GPS to atmospheric limb sounding and global monitoring. JPL Publ. 1994, 147, 94-18.

2. Kursinski, E.R.; Hajj, G.A.; Bertiger, W.I.; Leroy, S.S.; Meehan, T.K.; Romans, L.J; Schofield, J.T.; McCleese, D.J.; Melbourne, W.G.; Thornton, C.L.; et al. Initial results of radio occultation observations of Earth's atmosphere using the Global Positioning System. Science 1996, 271, 1107-1110. [CrossRef]

3. Steiner, A.K.; Kirchengast, G.; Ladreiter, H.-P. Inversion, error analysis, and validation of GPS/MET occultaiton data. Ann. Geophys. 1999, 17, 122-138. [CrossRef]

4. Yunck, T.P.; Hajj, G.A.; Kursinski, E.R.; LaBrecque, J.A.; Lowe, S.T.; Watkins, M.M.; McCormick, C. AMORE: An autonomous constellation concept for atmospheric and ocean observation. Acta Astronaut. 2000, 46, 355-364. [CrossRef]

5. Kirchengast, G.; Hoeg, P. The ACE+ mission: An atmosphere and climate explorer based on GPS, Galileo, and LEO-LEO radio occultation. In Occultations for Probing Atmosphere and Climate; Kirchengast, G., Foelsche, U., Steiner, A.K., Eds.; Springer: Berlin/Heidelberg, Germany, 2004; pp. 201-220.

6. Kursinski, E.R.; Feng, D.; Flittner, D.; Hajj, G.; Herman, B.; Romberg, F.; Syndergaard, S.; Ward, D.; Yunck, T. An active microwave limb sounder for profiling water vapor, ozone, temperature, geopotential, clouds, isotopes and stratospheric winds. In Occultations for Probing Atmosphere and Climate; Kirchengast, G., Foelsche, U., Steiner, A.K., Eds.; Springer: Berlin/Heidelberg, Germany, 2004; pp. 173-187. 
7. Kirchengast, G.; Schweitzer, S. Climate benchmark profiling of greenhouse gases and thermodynamic structure and wind from space. Geophys. Res. Lett. 2011, 38, 142-154. [CrossRef]

8. Proschek, V.; Kirchengast, G.; Schweitzer, S. Greenhouse gas profiling by infrared-laser and microwave occultation: Retrieval algorithm and demonstration results from end-to-end simulations. Atmos. Meas. Tech. 2011, 4, 2035-2058. [CrossRef]

9. Schweitzer, S.; Kirchengast, G.; Proschek, V. Atmospheric influences on infrared-laser signals used for occultation measurements between low Earth orbit satellites. Atmos. Meas. Tech. 2011, 4, 2273-2292. [CrossRef]

10. Brooke, J.S.A.; Bernath, P.F.; Kirchengast, G.; Thomas, C.B.; Wang, J.G.; Tereszchuk, K.A.; González Abad, G.; Hargreaves, R.J.; Beale, C.A.; Harrison, J.J.; et al. Greenhouse gas measurements over a $144 \mathrm{~km}$ open path in the Canary Islands. Atmos. Meas. Tech. 2012, 5, 2309-2319. [CrossRef]

11. Gurvich, A.S.; Gorbunov, M.E.; Fedorova, O.V.; Kirchengast, G.; Proschek, V.; Gonzalez Abad, G.; Tereszchuk, K.A. Spatiotemporal structure of a laser beam over $144 \mathrm{~km}$ in a Canary Islands experiment. Appl. Opt. 2012, 51, 7374-7383. [CrossRef]

12. Syndergaard, S.; Kirchengast, G. An Abel transform for deriving line-of-sight wind profiles from LEO-LEO infrared laser occultation measurements. J. Geophys. Res. Atmos. 2016, 121, 2525-2541. [CrossRef]

13. Anthes, R.A. Exploring Earth's atmosphere with radio occultation: Contributions to weather, climate and space weather. Atmos. Meas. Tech. 2011, 4, 1077-1103. [CrossRef]

14. Xu, X.; Han, Y.; Luo, J.; Wickert, J.; Asgarimehr, M. Seeking optimal GNSS radio occultation constellations using evolutionary algorithms. Remote Sens. 2019, 11,571. [CrossRef]

15. Kursinski, E.R.; Ward, D.; Otarola, A.; Frehlich, R.; Groppi, C.; Albanna, S.; Shein, M.; Bertiger, W.; Pickett, H.; Ross, M. The active temperature, ozone and moisture microwave spectrometer (ATOMMS). In New Horizons in Occultation Research: Studies in Atmosphere and Climate; Springer: Berlin/Heidelberg, Germany, 2009; pp. 295-313.

16. Kursinski, E.R.; Young, A.; Otarola, A.; Stovern, M.; Wheelwright, B.; Ward, D.; Sammler, K.; Stickney, R.; Groppi, C.; Banna, S.A.; et al. Laboratory and ground testing results from ATOMMS: The active temperature, ozone and moisture microwave spectrometer. In Proceedings of the 21st International Symposium on Space Terahertz Technology, Oxford, UK, 23-25 March 2010; pp. 155-163.

17. Kursinski, E.R.; Ward, D.; Stovern, M.; Otarola, A.C.; Young, A.; Wheelwright, B.; Stickney, R.; Albanna, S.; Duffy, B.; Groppi, C.; et al. Development and testing of the active temperature, ozone and moisture microwave spectrometer (ATOMMS) $\mathrm{cm}$ and $\mathrm{mm}$ wavelength occultation instrument. Atmos. Meas. Tech. 2012, 5, 439-456. [CrossRef]

18. Liu, C.L.; Kirchengast, G.; Syndergaard, S.; Kursinski, E.R.; Sun, Y.Q.; Bai, W.H.; Du, Q.F. A review of low Earth orbit occultation using microwave and infrared-laser signals for monitoring the atmosphere and climate. Adv. Space Res. 2017, 60, 2776-2811. [CrossRef]

19. Liu, C.; Kirchengast, G.; Sun, Y.; Wang, X.; Lu, D.; Bai, W.; Du, Q.; Loscher, A.; Syndergaard, S.; Tian, L.; et al. Exploring greenhouse gases water and climate changes: Scientific opportunities for the climate and atmospheric composition exploring satellites mission. Chin. J. Space Sci. 2021, 40, 151-168.

20. Harrison, J.J.; Bernath, P.F.; Kirchengast, G. Spectroscopic requirements for ACCURATE, a microwave and infrared-laser occultation satellite mission. J. Quant. Spectrosc. Radiat. Transf. 2011, 112, 2347-2354. [CrossRef]

21. Lackner, B.C.; Steiner, A.K.; Hegerl, G.C.; Kirchengast, G. Atmospheric climate change detection by radio occultation data using a fingerprinting method. J. Clim. 2011, 24, 5275-5291. [CrossRef]

22. Gurvich, A.S.; Gorbunov, M.E.; Fedorova, O.V.; Fortus, M.I.; Kirchengast, G.; Proschek, V.; Tereszchuk, K.A. Spatiotemporal structure of a laser beam at a path length of $144 \mathrm{~km}$ : Comparative analysis of spatial and temporal spectra. Appl. Opt. 2014, 53, 2625-2631. [CrossRef]

23. Proschek, V.; Kirchengast, G.; Schweitzer, S.; Brooke, J.S.A.; Bernath, P.F.; Thomas, C.B.; Wang, J.G.; Tereszchuk, K.A.; González Abad, G.; Hargreaves, R.J.; et al. Retrieval and validation of carbon dioxide, methane and water vapor for the Canary Islands ir-laser occultation experiment. Atmos. Meas. Tech. 2015, 8, 3315-3336. [CrossRef]

24. Fjeldbo, G.; Kliore, A.J. The neutral atmosphere of Venus as studied with the Marriner V radio occultation experiments. Astron. J. 1971, 76, 123-140. [CrossRef]

25. Liu, A.S. On the determination and investigation of the terrestrial ionospheric refractive indices using GEO-3/ATS-6 satellite-tosatellite tracking data. Radio Sci. 1978, 13, 709-716. [CrossRef]

26. Kursinski, E.R.; Hajj, G.A.; Schofield, J.T.; Linfield, R.P.; Hardy, K.R. Observing Earth's atmosphere with radio occultation measurements using the global positioning system. J. Geophys. Res. 1997, 102, 23429-23465. [CrossRef]

27. Wickert, J.; Reigber, C.; Beyerle, G.; Konig, R.; Marquardt, C.; Schmidt, T.; Grunwaldt, L.; Galas, R.; Meehan, T.K.; Melbourne, W.G.; et al. Atmosphere sounding by GPS radio occultation: First results from CHAMP. Geophys. Res. Lett. 2001, 28, 3263-3266. [CrossRef]

28. Foelsche, U.; Kirchengast, G.; Steiner, A.K. Global climate monitoring based on CHAMP/GPS radio occultation data. In First CHAMP Mission Results for Gravity, Magnetic and Atmospheric Studies; Springer: Berlin/Heidelberg, Germany, 2003 ; pp. 397-407.

29. Foelsche, U.; Pirscher, B.; Borsche, M.; Kirchengast, G.; Wickert, J. Assessing the climate monitoring utility of radio occultation data: From CHAMP to Formosat-3/COSMIC. Terr. Atmos. Ocean. Sci. 2009, 20, 155-170. [CrossRef]

30. Beyerle, G. GPS radio occultation with GRACE: Atmospheric profiling utilizing the zero difference technique. Geophys. Res. Lett. 2005, 32, L13806. [CrossRef] 
31. Wickert, J.; Schmidt, T.; Michalak, G.; Heise, S.; Arras, C.; Beyerle, G.; Falck, C.; König, R.; Pingel, D.; Rothacher, M. GPS radio occultation with CHAMP, GRACE-A, SAC-C, TerraSar-X, and Formosat-3/COSMIC: Brief review of results from GFZ. In New Horizons in Occultation Research; Steiner, A., Pirscher, B., Foelsche, U., Kirchengast, G., Eds.; Springer: Berlin/Heidelberg, Germany, 2009; pp. 3-15. [CrossRef]

32. Luntama, J.P.; Kirchengast, G.; Borsche, M.; Foelsche, U.; Steiner, A.; Healy, S.; von Engeln, A.; O'Clerigh, E.; Marquardt, C. Prospects of the EPS GRAS mission for operational atmospheric applications. Bull. Am. Meteorol. Soc. 2008, 89, $1863-1875$. [CrossRef]

33. Cucurull, L.; Kuo, Y.H.; Barker, D.; Rizvi, S.R.H. Assessing the impact of simulated COSMIC GPS radio occultation data on weather analysis over the antarctic: A case study. Mon. Weather. Rev. 2006, 134, 3283-3296. [CrossRef]

34. Schreiner, W.; Rocken, C.; Sokolovskiy, S.; Syndergaard, S.; Hunt, D. Estimates of the precision of GPS radio occultations from the COSMIC/Formosat-3 mission. Geophys. Res. Lett. 2007, 34, 545-559. [CrossRef]

35. Anthes, R.A.; Ector, D.; Hunt, D.C.; Kuo, Y.H.; Rocken, C.; Schreiner, W.S.; Sokolovskiy, S.V.; Syndergaard, S.; Wee, T.K.; Zeng, Z.; et al. The COSMIC/Formosat-3 mission: Early results. Bull. Am. Meteorol. Soc. 2008, 89, 313-333. [CrossRef]

36. Bai, W.H.; Liu, C.L.; Meng, X.G.; Sun, Y.Q.; Kirchengast, G.; Du, Q.F.; Wang, X.Y.; Yang, G.L.; Liao, M.; Yang, Z.D.; et al. Evaluation of atmospheric profiles derived from single- and zero-difference excess phase processing of BeiDou radio occultation data from the FY-3C GNOS mission. Atmos. Meas. Tech. 2018, 11, 819-833. [CrossRef]

37. Sun, Y.Q.; Bai, W.H.; Liu, C.L.; Liu, Y.; Du, Q.F.; Wang, X.Y.; Yang, G.L.; Liao, M.; Yang, Z.D.; Zhang, X.X.; et al. The FengYun-3C radio occultation sounder GNOS: A review of the mission and its early results and science applications. Atmos. Meas. Tech. 2018, 11, 5797-5811. [CrossRef]

38. Adhikari, L.; Ho, S.-P.; Zhou, X. Inverting COSMIC-2 phase data to bending angle and refractivity profiles using the full spectrum inversion method. Remote Sens. 2021, 13, 1793. [CrossRef]

39. Juang, J.-C.; Tsai, Y.-F.; Chu, C.-H. On constellation design of multi-GNSS radio occultation mission. Acta Astronaut. 2013, 82, 88-94. [CrossRef]

40. Bauer, P.; Radnóti, G.; Healy, S.; Cardinali, C. GNSS radio occultation constellation observing system experiments. Mon. Weather. Rev. 2014, 142, 555-572. [CrossRef]

41. Mousa, A.; Aoyama, Y.; Tsuda, T. A simulation analysis to optimize orbits for a tropical GPS radio occultation mission. Earth Planets Space 2006, 58, 919-925. [CrossRef]

42. Asgarimehr, M.; Hossainali, M.M. GPS radio occultation constellation design with the optimal performance in Asia Pacific region. J. Geod. 2015, 89, 519-536. [CrossRef]

43. Ghoniem, I.; Mousa, A.E.-K.; El-Fiky, G. Distribution of the GNSS-LEO occultation events over Egypt. NRIAG J. Astron. Geophys. 2019, 6, 97-103. [CrossRef]

44. Ghoniem, I.F.; Mousa, A.E.-K.; El-Fiky, G. GNSS-RO LEO satellite orbit optimization for Egypt and the middle east region. Alex. Eng. J. 2020, 59, 389-397. [CrossRef]

45. WATS-water vapor and temperature in the troposphere and stratosphere (3rd Report of Reports for mission selection, the five candidate Earth Explorer core missions). ESA Spec. Publ. 2001, 1257, 98.

46. Mimoun, D.; Abbondanza, S. The genesis of the ACE+ anti-rotating satellites concept. In Occultations for Probing Atmosphere and Climate; Springer: Berlin/Heidelberg, Germany; New York, NY, USA, 2004; pp. 245-258.

47. Kirchengast, G.; Ramsauer, J. Impact of an Atmospheric Profiling Mission on NWP: Assessment of Mission Scenarios and Definition of OSSE Study Test Missions. IMG/UoG Technical Report for ESA/ESTEC No. 3; 1999; 16p. Available online: https: //wegcwww.uni-graz.at/publ/users/gki/web/1999/gkandjr-imgtechrepfesa-16p-n3y1999.pdf (accessed on 23 November 2021).

48. Kornblueh, L.; Bengtsson, L.; Kirchengast, G.; Huang, X.-Y.; Hoeg, P.; Larsen, G.B. Impact of an Atmospheric Profiling Mission on Numerical Weather Prediction. 2000. Available online: https://wegcwww.uni-graz.at/publ/users/gki/web/arsclisys/ publications/publ2000/LKornbluehetal-ESAStudyRep-110p-y2000.pdf (accessed on 23 November 2021).

49. Kirchengast, G.; Bernath, P.; Buehler, S. ACCURATE-climate benchmark profiling of greenhouse gases and thermodynamic variables and wind from space (ESA Earth Explorer opportunity mission EE-8 proposal). Sci. Rep. 2010. Available online: https://wegccon.uni-graz.at/opac2010/pdf_presentation/opac_2010_kirchengast_gottfried_presentation57.pdf (accessed on 23 November 2021).

50. Kirchengast, G.; Zwanziger, C.; Larsen, G.B. Scientific Impact of an ACCURATE Mission and Synergies and Complementarities with Other Missions and GHG Observations; Technical Report for ESA-ESTEC No. 1/2010; 51p. Available online: https: //wegcwww.uni-graz.at/publ/wegcpubl/arsclisys/2010/wegc_gkirchengastetal-wegctechrepfesa-no1-2010.pdf (accessed on 23 November 2021).

51. Kursinski, E.R.; Folkner, W.; Zuffada, C.; Walker, C.; Hinson, D.; Ingersoll, A.; Gurwell, M.A.; Schofield, J.T.; Limaye, S.; Stern, A.; et al. The Mars atmospheric constellation observatory (MACO) concept. In Occultations for Probing Atmosphere and Climate; Kirchengast, G., Foelsche, U., Steiner, A.K., Eds.; Springer: Berlin/Heidelberg, Germany, 2004; pp. 393-405. [CrossRef]

52. Lee, S.; Mortari, D. 2-D lattice flower constellations for radio occultation missions. Front. Aerosp. Eng. 2013,2 , 79-90.

53. Vallado, D.; Crawford, P.; Huisak, R.; Kelso, T.S. Revisiting Spacetrack Report \#3. In Proceedings of the AIAA/AAS Astrodynamics Specialist Conference \& Exhibit, Keystone, CO, USA, 21-24 August 2006. [CrossRef]

54. Hofmann-Wellenhof, B.; Lichtenegger, H.; Wasle, E. GNSS—Global Navigation Satellite Systems; Springer: Berlin/Heidelberg, Germany; New York, NY, USA, 2008. 